\title{
GEOLOGICAL, GEOCHEMICAL, AND PETROGENETIC ASPECTS OF LATE NEOPROTEROZOIC YOUNGER GRANITES AT WADI UM SIDRA-WADI UM ASMER AREA, NORTH EASTERN DESERT, EGYPT.
}

\author{
El Afandy, A.H. ${ }^{1}$, El Kaliouby, B. A. ${ }^{2}$ Eliwa, H. A. ${ }^{3}$, Khamis, H. A. ${ }^{1}$, and Seddiek, S. H. ${ }^{1}$ \\ 1-Nuclear Materials Authority, P.O. 530, El-Maadi, Cairo, Egypt \\ 2- Geology Department, Faculty of Sience, Ain Shams University, Cairo, Egypt. \\ 3- Geology Department, Faculty of Sience, Menoufia University, Shebin El Kom, Egypt
}

\begin{abstract}
The late Neoproterozioc younger granites in the study area are classified into monzogranites, syenogranites and alkali feldspar granites according to the field, petrography and geochemical investigations. Monzogranites are mainly exposed along W. Um Sidra at G. Abu Mesaid in the eastern parts of the study area, intruding the Dokhan volcanic with sharp contacts. Syenogranites are exposed at G. El Shagola, G. Abu Harba, G Abu Mesaid, and some parts along W. Um Sidra intrude the metavolcanics and Dokhan volcanics with sharp contacts and contain enclaves of them. Alkali feldspar granites are exposed at G. El Shaylah, G. Urf El Eir, G. El Hurus, and the western exposures of G. Abu Harba intruding the metavolcanics and Dokhan volcanics with sharp and irregular contacts. The $\mathrm{K} / \mathrm{Rb}$, $\mathrm{Ba} / \mathrm{Rb}$ and $\mathrm{Rb} / \mathrm{Sr}$ ratios for the studied granites indicate that they were originated from highly differentiated magma and have been formed by partial melting of granitic crustal rocks. These granites were generated in possible extension-related environment and in a post-collision granites environment. The post-collision granites have calc-alkalic affinites, metaluminous to slightly peraluminous, and exhibit most of the characteristics of highly fractionated I-type granites derived from tonalites (intermediate magmas) of crustal sources. The studied monzogranites and syenogranites have generated at moderate/high water pressure in the $3-8 \mathrm{kbar}$ and temperatures of about $660^{\circ} \mathrm{C}-685^{\circ} \mathrm{C}$, while the alkali feldspar granites were crystallizated at low water vapor pressure about $1 \mathrm{kbar}$ and temperature abour 660 ${ }^{\circ} \mathrm{C}$., suggesting crystallization at relatively shallow depth between 20 and $30 \mathrm{~km}$. The crystal-liquid equilibrium was the dominant mechanism involved in the genesis of these granites. The monzogranites and syenogranites have been generated at greater depth $>30 \mathrm{~km}$ of the lower crust. The overall decreasing trends in $\mathrm{FeO}^{\mathrm{t}}, \mathrm{MgO}, \mathrm{CaO}, \mathrm{TiO}_{2}$, and $\mathrm{P}_{2} \mathrm{O}_{5}$ with increasing $\mathrm{SiO}_{2}$ coupled with increasing $\mathrm{Rb}$, $\mathrm{Y}, \mathrm{Th}$ and $\mathrm{Nb}$ and the fractionated nature of the REE $\left[(\mathrm{La} / \mathrm{Lu})_{\mathrm{n}}=3.67-14.21\right]$ suggesting that the fractional crystallization was played the major role during the evolution of the studied granites.
\end{abstract}

Keywords: Neoproterozioc; monzogranites; syenogranites; alkali feldspar granites; Um Sidra; Abu Harba

\section{INTRODUCTION}

The distribution and volume of granitoids dominating the northern Arabian Nubian Shield (ANS) crust including the Eastern Desert (ED), is ubiquitous in the shield, (Farahat et al., 2007, 2011; El-Bialy and Omar, 2015). Within the Egyptian part, the dominance of granitoids increases northward attaining the highest abundance $(\sim 70 \%$ of the overall basement area) in Sinai, and even in the entire ANS (Bentor, 1985; El-Bialy and Streck, 2009). In the Egyptian basement, the relative abundance of the younger granite to the older granite increases from 1 to 4 in the southern ED to nearly 1 to 1 in the north (Stern, 1979) and 12 to 1 in Sinai (Bentor, 1985), in parallel with the general ANS trend of increasing granitoid abundance from south to north. Currently, it is well established that participation of pre-Neoproterozoic crust in the formation of the Older and Younger Granite groups was trivial (e.g. Hargrove et al., 2006; Moussa et al., 2008; Ali et al., 2009a,b, 2012; Ali, 2015). Nevertheless, there is no concurrence in regard to other important petrogenetic concerns, including (a) their source, (b) their regional geotectonic regimes, and (c) their peculiar individual petrogenetic paths. Therefore, different workers maintained that one group, or both groups, evolved by partial melting of lower/middle crustal materials or instead by fractionation of 


\section{El Afandy, et al.}

mantle-derived melts. (E.g., El-Sayed and Nisr, 1999; Moghazi et al., 2004; Moghazi, 2002; Farahat et al., 2011; Ali et al., 2012, 2013, 2016; El Mahallawi and Ahmed, 2012; Azer, 2013; Eliwa et al., 2014; Mahdy et al., 2015; El-Bialy and Omar, 2015; Asran et al., 2017; Sami et al.,2018; El-Bialy and Shata, 2018).

Hussein et al. (1982) divided the Egyptian younger granites into suture-related granites (G2-granite) formed in post-orogenic environment and intraplate granites (G3-granite) related to rifting processes. Hassan and Hashad (1990) suggested that the magma of the younger granites was emplaced in three possible tectonic settings: (1) subduction processes in a volcanic arc environment, (2) arc-continent collision event, and (3) within continental plates. Noweir et al. (1990) considered the Egyptian younger granites as transitional phases from calc-alkaline I-type magmatism to normal alkaline and peralkaline Atype granite. EI-Sayed (1998) classified the Egyptian granites into I-type orogenic arc related and A-type anorogenic rift-related granites.

In this paper, we present comprehensive field and petrographic obserevations and whole-rock geochemistry data for the exposed younger granite at the areas surrounding Wadi Um Sidra and Wadi Um Asmer (NED) of Egypt in order to detect their origin, tectonic setting and the thermo-barometric conditions of crystallization.

\section{GEOLOGIC SETTING AND PETROGRAPHY}

The investigated area is located at about $50 \mathrm{~km}$ northwest of Hurghada city in the NED of Egypt between Latitudes $27^{\circ} 14^{\prime}$ and $27^{\circ} 26^{\prime} \mathrm{N}$ and Longitudes $33^{\circ} 8^{\prime}$ and $33^{\circ} 22^{\prime} \mathrm{E}$. The younger granites in the studied area could be distinguished into monzogranite, syenogranite and alkali feldspar granite (Fig.1).

Fig.1: Geological map of the Wadi Um Sidra and Wadi Um Asmer area, NED, Egypt.

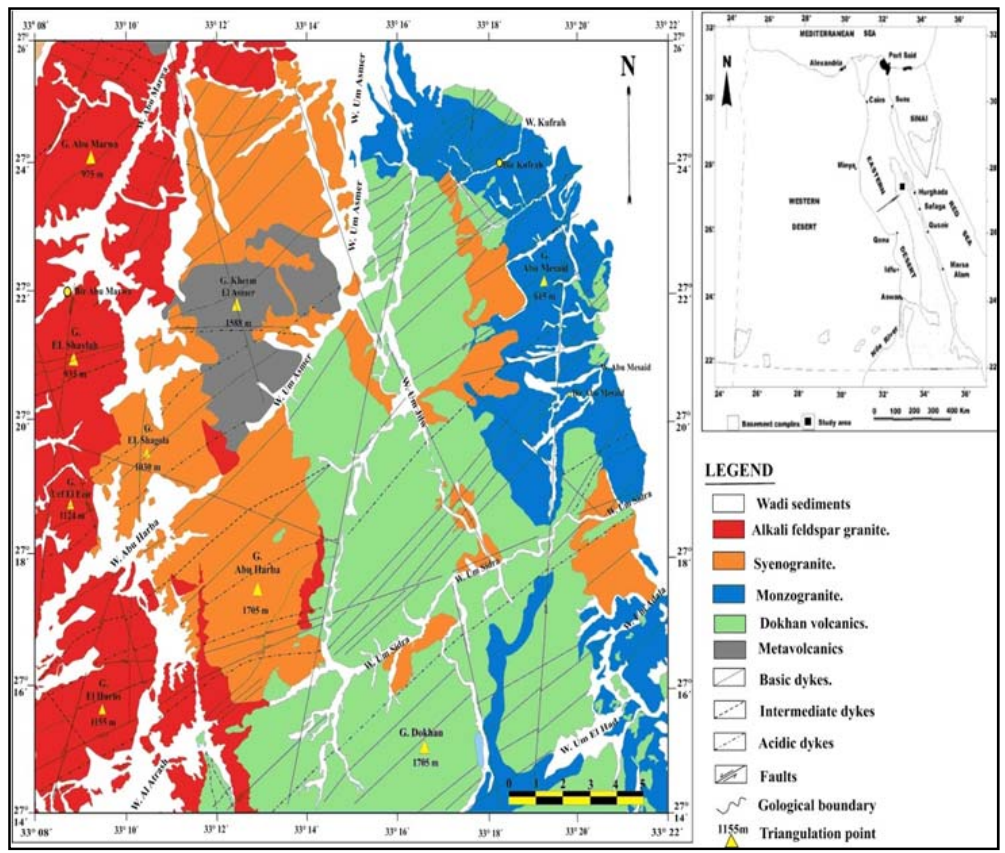

Monzogranites are mainly exposed along Wadi Um Sidra at Gebel Abu Mesaid in the eastern parts of the area (Fig. 1). These rocks are generally form low to moderate topographic reliefs. They are jointed and dissected by numerous faults and shear zones. The contact between the Dokhan volcanics and these granites is rather sharp intrusive and chilled. Monzogranites intrude the Dokhan volcanics and send several offshoots into them. These rocks contain xenoliths of different shapes and sizes of Dokhan volcanics. The monzogranites are medium to coarse-grained and porphyritic varieties are common. They are blocky with greyish pink color, and show hypidiomorphic equigranular texture. They are composed of potash-feldspar (orthoclase and microcline perthite), plagioclases $\left(\mathrm{An}_{14-29}\right)$, quartz, biotite and hornblende (Fig.2a) as essential minerals. Opaque minerals, sphene, allanite, zircon and apatite are the accessory minerals while epidote and chlorite are secondary minerals. 


\section{Geological, geochemical, and petrogenetic aspects}

The syenogranites represent the predominant granitic rock in the area. They are mainly exposed at G. El Shagola, Abu Harba, Abu Mesaid, and some parts along W. Um Sidra, (Fig. 1). They form moderate to very high rugged serrated peaks with smooth slopes. They are highly sheared and weathered, intruding the metavolcanics and Dokhan volcanics with sharp contacts and contains enclaves of them. They are traversed by dyke swarms of various trends, compositions, and thickness. The syenogranites in the area are medium to coarse-grained and pinkish white in color. They are generally equigranular (sometimes porphyritic) with hypidiomorphic textures, composed of potash feldspar (orthoclase and microcline perthites), quartz, plagioclase $\left(\mathrm{An}_{8-20}\right)$ and biotite as essential minerals. Accessories include zircon, sphene, muscovite, apatite and iron oxides (Fig. 2b), while epidote and chlorite are secondary minerals.

The alkali feldspar granites cover significant surface exposures in the western part of the study area. These granites are exposed at G. El Shaylah, G. Urf El Eir, G. El Hurus, and the western exposures of G. Abu Harba, (Fig. 1). They form series of intrusive granitic masses of moderate to highly elevated mountains with prominent ridges and steep slopes. They intrude the metavolcanics and Dokhan volcanics with sharp and irregular contacts. The alkali feldspar granites are medium to coarse-grained, pink to pinkish red in color. The most characteristic feature of these granites are the deficiency in the ferromagnesian minerals, where some samples appear to be free of them, giving rise to a leucocratic type. Microscopically, these rocks display hybidiomorphic equigranular texture and mainly composed of potash feldspar (orthoclase and microcline perthites), quartz, plagioclases $\left(\mathrm{An}_{5-15}\right)$ and few biotite (Fig .2d) as essentially minerals. The main accessory minerals are zircon, sphene, apatite, allanite and iron oxides (Fig. 2 e\&f), while Muscovite, chlorite, sericite and epidote are secondary minerals.

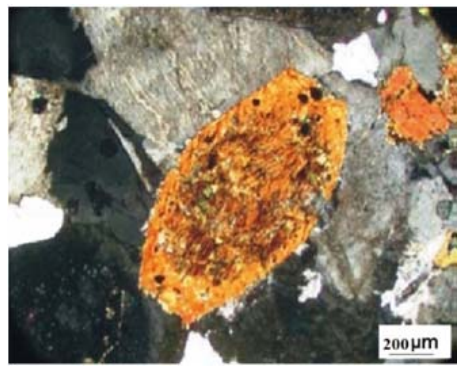

a

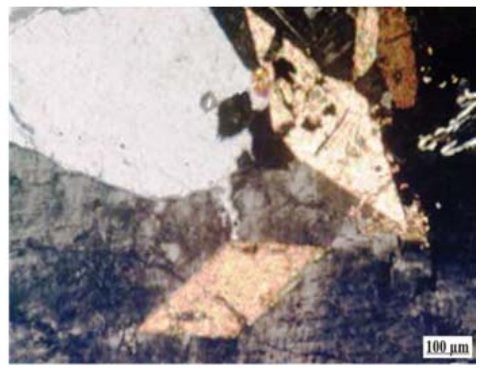

b

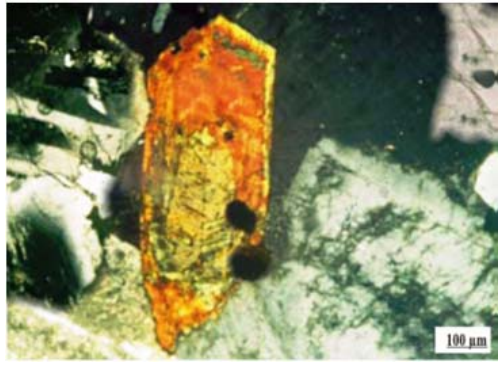

c

Fig. 2: Photomicrographs of younger granites: a) Euhedral hornblende crystal enclosing fine quartz grains, and showing alteration to biotite, and opaques in monzogranite C.N; b) Wedge-shaped sphene crystals with fine zircon and opaques enclosed within microcline perthite, in syenogranite C.N.; c) Suhedral prismatic allanite showing zonation and enclosing opaques in alkali feldspar granite, C.N.

\section{GEOCHEMISTRY}

The studied granites were chemically analyzed for major oxides and trace elements using Rigaku RIX 2000 X-ray fluorescence (XRF) spectrometer. Selected samples were analyzed for rare earth elements using inductively coupled plasma- mass spectrometry (ICP-MS). All analyses were carried out at the Department of Earth Sciences, Faculty of Science, Naruto University, Japan. The results of major oxides and trace elements with computed CIPW norms as well as some calculated parameters are listed in Table 1, while rare earth elements (REEs) are listed in Table 2.

\section{Geochemical classification}

On the total alkalis vs. silica (TAS) diagram of Middlemost (1985), (Fig. 3), the monzogranite and syenogranite samples plot within the granite field whereas the alkali feldspar granite samples fall within the field of alkali feldspar granite. Plotting the studied granites in the classification diagram of De La Roche et al. (1980), (Fig. 4), they fall consistently within the monzogranite, syanogranite and alkali feldspar granite fields, in a complete harmony with petrographic studies.

Some significant trace elements (ppm) are plotted against $\mathrm{SiO}_{2}$ (Fig. 5). The concentrations of $\mathrm{Sr}, \mathrm{Ba}$, $\mathrm{Y}$ and $\mathrm{Zr}$ decrease with the increase of $\mathrm{SiO}_{2}$ from monzogranites to alkali feldspar granites. Whereas $\mathrm{Rb}$, $\mathrm{Nb}$ and $\mathrm{Th}$ increase with increasing silica. The decrease of $\mathrm{Sr}, \mathrm{Ba}$ and $\mathrm{Zr}$ may be related to fractional 


\section{El Afandy, et al.}

crystallization of biotite, plagioclase and zircon (Jung et al., 2000; Moghazi, 2002). According to the alumina saturation index (ASI) of Zen (1986), the studied granites are mainly metaluminous to slightly peraluminous with $\mathrm{A} / \mathrm{CNK}$ values mostly clustering around narrow range of 0.90 to 1.09 (Table 1).

\section{Rare earth elements (REEs)}

Primitive mantle-normalized spider diagram (Sun and McDonough, 1989) for the studied granites show higher than primitive mantle values among the most elements (Fig. 6). The patterns of the monzogranites and syenogranites are very similar and show negative Ti and $\mathrm{P}$ anomalies and moderate negative $\mathrm{Sr}, \mathrm{Ba}$ and $\mathrm{Nb}$ anomalies. The alkali feldspar granites exhibit moderate/strong negative $\mathrm{Ba}, \mathrm{P}, \mathrm{Sr}$, and $\mathrm{Ti}$ anomalies and moderate negative $\mathrm{Nb}$. The common negative anomalies among all granites types are $\mathrm{P}, \mathrm{Sr}$ and Ti elements, probably attributed to the fractionation of apatite, plagioclase and Fe-Ti oxides (Brown et al., 1984).

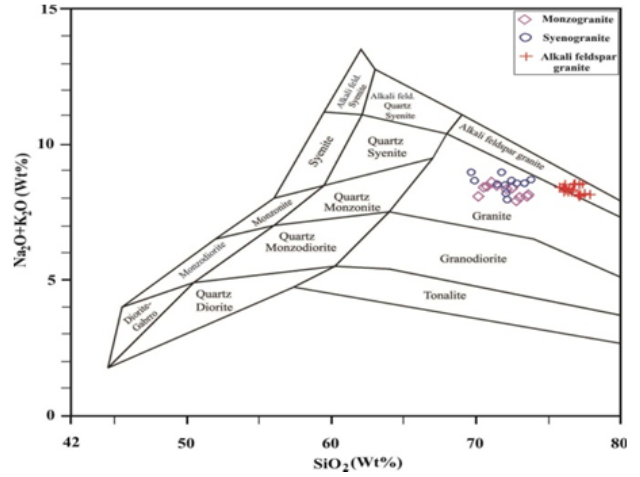

Fig. 3: Plots of the studied granites on the TAS classification diagram (Middlemost, 1985).

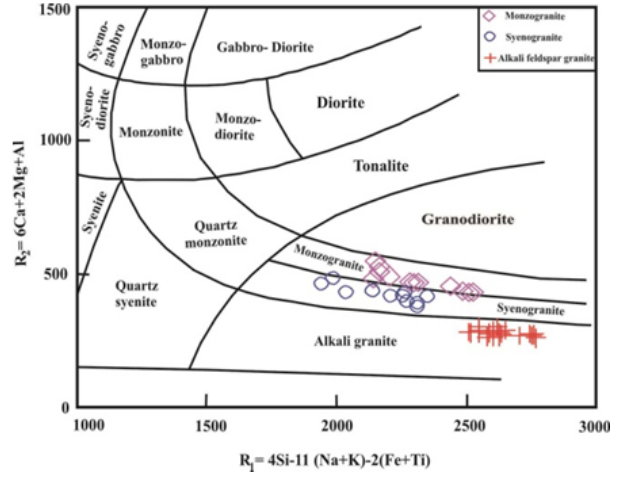

Fig. 4: Plots of the studied granites on R1 vs R2 classification diagram (De La Roche et al., 1982).
Fig. 5: Silica variation diagrams demonstrating concentration and compositional trends of selected trace elements (ppm) in the studied granites.
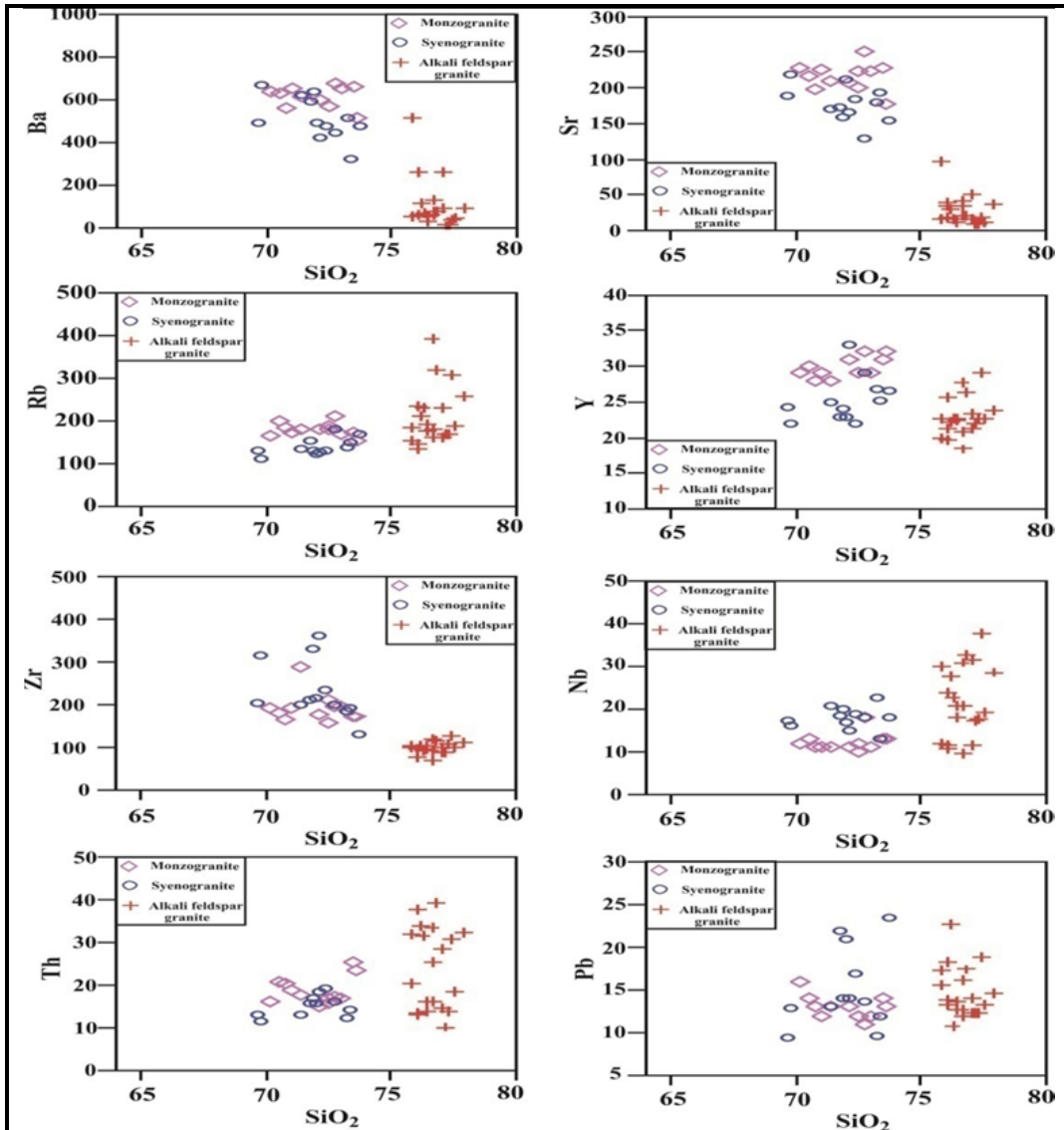

300 
Geological, geochemical, and petrogenetic aspects

Table 1: Major oxides, trace elements and CIPW norm, with some parameters, and geochemical ratios for the studied granites.

\begin{tabular}{|c|c|c|c|c|c|c|c|c|c|c|c|c|c|c|c|c|c|c|c|c|c|c|c|}
\hline \multirow{2}{*}{$\begin{array}{r}\text { Rock type } \\
\text { Sample No. }\end{array}$} & \multicolumn{3}{|c|}{ Monzogranite } & \multirow[b]{2}{*}{ GA-4 } & \multirow{2}{*}{ GA-5 } & \multirow{2}{*}{ GA-6 } & \multirow{2}{*}{ GA-7 } & \multirow{2}{*}{ GA-8 } & \multirow{2}{*}{ GA-9 } & \multirow{2}{*}{ GA-10 } & \multirow{2}{*}{ GA-11 } & \multirow{2}{*}{ GA-12 } & \multirow[b]{2}{*}{ Av. } & \multirow{2}{*}{\multicolumn{6}{|c|}{ 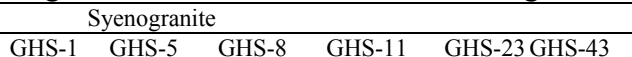 }} & \multirow{3}{*}{$\begin{array}{c}\text { GO-4 } \\
73.27\end{array}$} & \multirow{3}{*}{$\begin{array}{c}\text { GO-5 } \\
73.33\end{array}$} & & \\
\hline & GA-1 & GA-2 & GA-3 & & & & & & & & & & & & & & & & & & & & $\begin{array}{ll}\mathrm{GO}-7 \\
\end{array}$ \\
\hline $\mathrm{SiO}_{2}$ & 70.53 & 71.08 & 72.45 & 72.98 & 72.80 & 71.44 & 72.12 & 72.47 & 70.76 & 73.58 & 73.49 & 70.15 & 71.99 & 71.94 & 73.76 & 72.13 & 71.43 & 71.71 & 72.38 & & & 72.77 & 72.01 \\
\hline $\mathrm{TiO}_{2}$ & 0.42 & 0.36 & 0.29 & 0.29 & 0.33 & 0.40 & 0.30 & 0.30 & 0.38 & 0.28 & 0.24 & 0.36 & 0.33 & 0.41 & 0.31 & 0.51 & 0.48 & 0.40 & 0.36 & 0.23 & 0.24 & 0.35 & 0.37 \\
\hline $\mathrm{Al}_{2} \mathrm{O}_{3}$ & 14.24 & 14.13 & 13.96 & 13.88 & 14.06 & 14.28 & 13.98 & 13.85 & 14.14 & 13.48 & 13.77 & 14.30 & 14.01 & 13.51 & 12.88 & 13.45 & 13.44 & 13.84 & 13.19 & 12.69 & 12.61 & 13.10 & 13.39 \\
\hline $\mathrm{Fe}_{2} \mathrm{O}_{3}$ & 2.84 & 2.75 & 2.46 & 2.37 & 2.48 & 2.36 & 2.47 & 2.54 & 2.75 & 2.11 & 2.29 & 2.76 & 2.52 & 2.57 & 2.04 & 2.64 & 2.57 & 2.56 & 2.58 & 2.19 & 2.31 & 2.65 & 2.85 \\
\hline $\mathrm{MnO}$ & 0.05 & 0.05 & 0.05 & 0.06 & 0.06 & 0.05 & 0.06 & 0.05 & 0.06 & 0.06 & 0.05 & 0.07 & 0.06 & 0.09 & 0.05 & 0.06 & 0.10 & 0.09 & 0.09 & 0.07 & 0.08 & 0.08 & 0.08 \\
\hline $\mathrm{MgO}$ & 0.46 & 0.56 & 0.41 & 0.44 & 0.48 & 0.60 & 0.45 & 0.43 & 0.56 & 0.31 & 0.34 & 0.54 & 0.47 & 0.62 & 0.38 & 0.56 & 0.60 & 0.57 & 0.53 & 0.46 & 0.36 & 0.44 & 0.54 \\
\hline $\mathrm{CaO}$ & 1.82 & 1.62 & 1.34 & 1.41 & 1.25 & 1.67 & 1.34 & 1.43 & 1.86 & 1.16 & 1.19 & 2.14 & 1.52 & 1.33 & 0.98 & 1.37 & 1.34 & 1.25 & 1.28 & 1.16 & 1.17 & 1.31 & 1.36 \\
\hline $\mathrm{Na}_{2} \mathrm{O}$ & 3.95 & 4.14 & 4.16 & 3.85 & 3.60 & 4.10 & 3.91 & 4.04 & 4.12 & 3.52 & 3.51 & 4.59 & 3.96 & 4.83 & 4.19 & 4.15 & 4.52 & 4.72 & 4.25 & 4.42 & 4.15 & 4.22 & 4.34 \\
\hline $\mathrm{K}_{2} \mathrm{O}$ & 4.48 & 4.46 & 4.24 & 4.21 & 4.31 & 4.39 & 4.45 & 4.35 & 4.32 & 4.63 & 4.56 & 3.49 & 4.32 & 3.69 & 4.52 & 3.83 & 4.03 & 4.25 & 4.42 & 4.17 & 4.43 & 4.36 & 3.88 \\
\hline $\mathrm{P}_{2} \mathrm{O}_{5}$ & 0.12 & 0.08 & 0.07 & 0.07 & 0.08 & 0.10 & 0.07 & 0.07 & 0.11 & 0.06 & 0.07 & 0.12 & 0.09 & 0.12 & 0.05 & 0.08 & 0.11 & 0.10 & 0.09 & 0.05 & 0.05 & 0.08 & 0.12 \\
\hline L.O.I & 0.76 & 0.61 & 0.56 & 0.41 & 0.53 & 0.60 & 0.74 & 0.46 & 0.84 & 0.79 & 0.46 & 1.17 & 0.66 & 0.84 & 0.76 & 1.15 & 1.24 & 0.44 & 0.78 & 1.25 & 1.16 & 0.58 & 0.94 \\
\hline Total $\%$ & 99.67 & 99.84 & 99.99 & 99.97 & 99.98 & 99.99 & 99.89 & 99.99 & 99.90 & 99.98 & 99.97 & 99.69 & 99.91 & 99.95 & 99.92 & 99.93 & 99.86 & 99.93 & 99.95 & 99.96 & 99.89 & 99.94 & 99.88 \\
\hline Q & 26.2 & 26 & 28.7 & 30.9 & 32.1 & 26.6 & 28.9 & 28.7 & 26 & 32.5 & 32.7 & 24.7 & 28.65 & 26.56 & 29.86 & 29.71 & 26.52 & 25.02 & 27.83 & 29.16 & 29.90 & 28.70 & 28.57 \\
\hline $\mathrm{C}$ & 0.00 & 0.00 & 0.23 & 0.52 & 1.32 & 0.00 & 0.39 & 0.00 & 0.00 & 0.65 & 0.99 & 0.00 & 0.34 & 0.00 & 0.00 & 0.13 & 0.00 & 0.00 & 0.00 & 0.00 & 0.00 & 0.00 & 0.00 \\
\hline Or & 26.47 & 26.36 & 25.06 & 24.88 & 25.47 & 25.94 & 26.30 & 25.71 & 25.53 & 27.36 & 26.95 & 20.62 & 25.55 & 21.81 & 26.71 & 22.63 & 23.81 & 25.11 & 26.12 & 24.64 & 26.18 & 25.76 & 22.93 \\
\hline $\mathrm{Ab}$ & 33.42 & 35.03 & 35.20 & 32.58 & 30.46 & 34.69 & 33.08 & 34.18 & 34.86 & 29.78 & 29.70 & 38.84 & 33.49 & 40.87 & 35.45 & 35.12 & 38.25 & 39.94 & 35.96 & 37.40 & 35.12 & 35.71 & 36.72 \\
\hline An & 7.89 & 6.80 & 6.42 & 6.77 & 5.88 & 7.60 & 6.41 & 6.81 & 7.33 & 5.57 & 5.64 & 8.11 & 6.77 & 4.29 & 2.99 & 6.41 & 4.48 & 4.03 & 3.86 & 2.47 & 2.70 & 3.93 & 5.60 \\
\hline $\mathrm{Di}$ & 0.00 & 0.00 & 0.00 & 0.00 & 0.00 & 0.00 & 0.00 & 0.00 & 0.27 & 0.00 & 0.00 & 0.76 & 0.09 & 0.49 & 0.6 & 0.00 & 0.27 & 0.51 & 0.90 & 2.02 & 1.86 & 0.99 & 0.00 \\
\hline Hy & 1.15 & 1.39 & 1.02 & 1.10 & 1.20 & 1.49 & 1.12 & 1.07 & 1.30 & 0.77 & 0.85 & 0.78 & 1.10 & 1.32 & 0.65 & 1.39 & 1.37 & 1.18 & 0.90 & 0.21 & 0.04 & 0.64 & 1.34 \\
\hline $\mathrm{Hm}$ & 2.84 & 2.75 & 2.46 & 2.37 & 2.48 & 2.36 & 2.47 & 2.54 & 2.75 & 2.11 & 2.29 & 2.76 & 2.52 & 2.57 & 2.04 & 2.64 & 2.57 & 2.56 & 2.58 & 2.19 & 2.31 & 2.65 & 2.85 \\
\hline Il & 0.11 & 0.11 & 0.10 & 0.13 & 0.13 & 0.11 & 0.13 & 0.11 & 0.13 & 0.13 & 0.11 & 0.15 & 0.12 & 0.19 & 0.11 & 0.13 & 0.21 & 0.19 & 0.19 & 0.15 & 0.17 & 0.17 & 0.17 \\
\hline Ap & 0.28 & 0.19 & 0.17 & 0.17 & 0.19 & 0.24 & 0.17 & 0.17 & 0.26 & 0.14 & 0.17 & 0.28 & 0.20 & 0.28 & 0.12 & 0.19 & 0.26 & 0.24 & 0.21 & 0.12 & 0.12 & 0.19 & 0.28 \\
\hline DI & 85.8 & 87.3 & 88.9 & 88.3 & 88 & 87.2 & 88.3 & 88.6 & 85.8 & 89.7 & 89.3 & 85 & 87.68 & 89.24 & 92.02 & 87.46 & 88.58 & 90.07 & 89.91 & 91.20 & 91.20 & 90.17 & 88.22 \\
\hline $\mathrm{AI}$ & 0.80 & 0.82 & 0.82 & 0.78 & 0.75 & 0.81 & 0.80 & 0.82 & 0.81 & 0.80 & 0.78 & 0.80 & 0.80 & 0.88 & 0.91 & 0.82 & 0.88 & 0.89 & 0.89 & 0.93 & 0.92 & 0.89 & 0.85 \\
\hline ASI & 0.97 & 0.97 & $\begin{array}{l}1.01 \\
\text { l }\end{array}$ & 1.03 & 1.09 & 0.98 & $\begin{array}{l}1.02 \\
1.02\end{array}$ & 0.99 & 0.95 & $\begin{array}{l}1.04 \\
\text { lat }\end{array}$ & 1.07 & 0.94 & 1.01 & 0.94 & 0.95 & 1.00 & 0.94 & 0.95 & 0.94 & 0.91 & 0.92 & 0.93 & 0.97 \\
\hline $\mathrm{AR}$ & 3.21 & 3.41 & 3.43 & 3.23 & 3.14 & 3.28 & 3.4 & 3.44 & 3.23 & 3.51 & 3.34 & 2.93 & 3.30 & 3.70 & 4.38 & 3.33 & 3.74 & 3.93 & 3.99 & 4.27 & 4.30 & 3.94 & 3.52 \\
\hline Mg\# & 24.3 & 28.7 & 24.8 & 26.9 & 27.7 & 33.5 & 26.5 & 25.1 & 28.7 & 22.5 & 22.7 & 27.9 & 26.61 & 32.3 & 27.0 & 29.6 & 31.6 & 30.6 & 28.9 & 29.4 & 23.6 & 24.8 & 27.3 \\
\hline $\mathrm{Ba}$ & 629 & 652 & 644 & 656 & 676 & 613 & 600 & 569 & 558 & 516 & 657 & 639 & 617 & 637 & 478 & 420 & 621 & 590 & 479 & 514 & 322 & 446 & 494 \\
\hline $\mathrm{Rb}$ & 135 & 134 & 149 & 123 & 156 & 141 & 132 & 127 & 124 & 119 & 121 & 135 & 133 & 132 & 167 & 125 & 135 & 154 & 131 & 137 & 149 & 180 & 121 \\
\hline $\mathrm{Sr}$ & 216 & 225 & 223 & 224 & 251 & 210 & 207 & 200 & 197 & 177 & 227 & 228 & 216 & 159 & 155 & 165 & 171 & 172 & 184 & 179 & 192 & 130 & 212 \\
\hline $\mathrm{Nb}$ & 13 & 11 & 12 & 11 & 18 & 11 & 11 & 10 & 11 & 13 & 13 & 12 & 12 & 20 & 18 & 15 & 21 & 18 & 19 & 23 & 13 & 18 & 17 \\
\hline $\mathrm{Y}$ & 30 & 29 & 29 & 29 & 32 & 28 & 31 & 29 & 28 & 32 & 31 & 29 & 30 & 24 & 27 & 33 & 25 & 23 & 22 & 27 & 25 & 29 & 23 \\
\hline $\mathrm{Zr}$ & 180 & 192 & 210 & 196 & 196 & 289 & 177 & 159 & 164 & 171 & 174 & 193 & 192 & 329 & 131 & 361 & 199 & 210 & 233 & 184 & 192 & 201 & 213 \\
\hline $\mathrm{Ce}$ & 60 & 64 & 69 & 55 & 79 & 70 & 59 & 51 & 57 & 63 & 58 & 56 & 62 & 75 & 50 & 77 & 68 & 69 & 70 & 56 & 57 & 48 & 69 \\
\hline $\mathrm{Cr}$ & 10 & 8 & 13 & 6 & 14 & 5 & 8 & 5 & 12 & 7 & 7 & 16 & 9 & 11 & 6 & 22 & 10 & 10 & 16 & 13 & 8 & 6 & 19 \\
\hline $\mathrm{Ni}$ & 1 & 1 & 2 & 2 & 3 & 0 & 1 & 1 & 2 & 2 & 2 & 9 & 2 & nd & nd & nd & nd & 1 & nd & nd & nd & nd & nd \\
\hline $\mathrm{Pb}$ & 14 & 12 & 12 & 12 & 11 & 13 & 13 & 12 & 13 & 13 & 14 & 16 & 13 & 14 & 23 & 14 & 13 & 22 & 17 & 10 & 12 & 14 & 21 \\
\hline Th & 20.7 & 18.8 & 15.9 & 16.8 & 17.3 & 17.8 & 14.9 & 17.1 & 20.3 & 23.4 & 25.4 & 16.2 & 18.7 & 16.8 & 31.2 & 18.4 & 13.2 & 15.8 & 19.2 & 12.4 & 14.4 & 16.1 & 15.9 \\
\hline U & 5.3 & 5.1 & 4.3 & 4.1 & 4.3 & 4.1 & 4.6 & 4.2 & 5.2 & 7.2 & 9.8 & 3.6 & 5.1 & 4.3 & 9.2 & 4.9 & 4.1 & 5.7 & 6.6 & 4.1 & 4.2 & 4.2 & 5.6 \\
\hline $\mathrm{K} / \mathrm{Rb}$ & 275.5 & $276 . .3$ & 236.2 & 284.2 & 229.4 & 258.5 & 279.9 & 284.4 & 289.2 & 323.0 & 312.9 & 227.5 & 273.08 & 232.9 & 224.3 & 254.4 & 247.8 & 228.6 & 280.7 & 253.4 & 247.2 & 200.6 & 266.2 \\
\hline $\mathrm{Ba} / \mathrm{Rb}$ & 4.66 & 4.87 & 4.32 & 5.33 & 4.33 & 4.35 & 4.55 & 4.48 & 4.50 & 4.34 & 5.43 & 4.73 & 4.66 & 4.84 & 2.86 & 3.36 & 4.60 & 3.82 & 3.66 & 3.76 & 2.16 & 2.47 & 4.08 \\
\hline $\mathrm{Rb} / \mathrm{Sr}$ & 0.62 & 0.60 & 0.67 & 0.55 & 0.62 & 0.67 & 0.64 & 0.63 & 0.63 & 0.67 & 0.53 & 0.59 & 0.62 & 0.83 & 1.08 & 0.76 & 0.79 & 0.90 & 0.71 & 0.76 & 0.77 & 1.39 & 0.57 \\
\hline
\end{tabular}

D.I: Differentiation Index; AI: Agpaitic Index; ASI: Alumina Saturation Index; AR: Alkalinity Ratio: $\mathrm{Mg} \#=\mathrm{Mg} \times 100 /\left(\mathrm{Mg}+\mathrm{Fe}^{2}\right)$. 
El Afandy, et al.

Table 1: (Continued)

\begin{tabular}{|c|c|c|c|c|c|c|c|c|c|c|c|c|c|c|c|c|c|c|c|c|c|c|c|c|}
\hline \multicolumn{2}{|l|}{ Rock type } & \multicolumn{2}{|c|}{\begin{tabular}{|l|l} 
Syenogranite \\
\end{tabular}} & \multicolumn{5}{|c|}{ Alkali feldspar granite } & \multirow[b]{2}{*}{ GH-6 } & \multirow[b]{2}{*}{ GH-7 } & \multirow[b]{2}{*}{ GH-8 } & \multirow[b]{2}{*}{ GH-9 } & \multirow[b]{2}{*}{ GH-10 } & \multirow[b]{2}{*}{ GSH-1 } & \multirow[b]{2}{*}{ GSH-2 } & \multirow[b]{2}{*}{ GSH-3 } & \multirow[b]{2}{*}{ GSH-4 } & \multirow[b]{2}{*}{ GSH-5 } & \multirow[b]{2}{*}{ GSH-6 } & \multirow[b]{2}{*}{ GSH-7 } & & & & \\
\hline & GO-8 & GO-11 & Av. & GH-1 & GH-2 & $\mathrm{GH}-3$ & GH-4 & GH-5 & & & & & & & & & & & & & GSH-8 & GSH-9 & GSH-10 & Av. \\
\hline $\mathrm{SiO}_{2}$ & 69.63 & 69.84 & 72.02 & 75.77 & 76.73 & 76.15 & 76.25 & 77.10 & 76.86 & 77.36 & 76.62 & 77.91 & 76.04 & 77.50 & 76.48 & 76.39 & 77.24 & 77.09 & 77.16 & 76.11 & 76.10 & 75.79 & 76.68 & 76.67 \\
\hline $\mathrm{TiO}_{2}$ & 0.53 & 0.62 & 0.40 & 0.21 & 0.11 & 0.10 & 0.10 & 0.09 & 0.10 & 0.11 & 0.11 & 0.12 & 0.11 & 0.13 & 0.15 & 0.15 & 0.11 & 0.11 & 0.11 & 0.12 & 0.10 & 0.14 & 0.11 & 0.12 \\
\hline $\mathrm{Al}_{2} \mathrm{O}_{3}$ & 13.93 & 14.29 & 13.36 & 12.18 & 12.10 & 12.18 & 12.07 & 11.92 & 11.86 & 11.68 & 12.11 & 11.53 & 12.34 & 11.79 & 11.90 & 11.83 & 11.63 & 11.89 & 11.67 & 12.15 & 12.13 & 12.35 & 12.26 & 11.98 \\
\hline $\mathrm{Fe}_{2} \mathrm{O}_{3}$ & 2.97 & 2.98 & 2.58 & 2.01 & 1.51 & 1.73 & 1.86 & 1.38 & 1.48 & 1.31 & 1.48 & 1.21 & 1.46 & 1.31 & 1.83 & 1.74 & 1.48 & 1.38 & 1.39 & 1.62 & 1.61 & 1.94 & 1.38 & 1.56 \\
\hline $\mathrm{MnO}$ & 0.09 & 0.09 & 0.08 & 0.04 & 0.05 & 0.04 & 0.03 & 0.03 & 0.03 & 0.06 & 0.05 & 0.02 & 0.06 & 0.04 & 0.05 & 0.04 & 0.05 & 0.03 & 0.04 & 0.05 & 0.04 & 0.04 & 0.05 & 0.04 \\
\hline $\mathrm{MgO}$ & 0.70 & 0.74 & 0.54 & 0.15 & 0.10 & 0.06 & 0.06 & 0.05 & 0.08 & 0.10 & 0.11 & 0.07 & 0.06 & 0.09 & 0.09 & 0.09 & 0.07 & 0.08 & 0.06 & 0.09 & 0.08 & 0.04 & 0.10 & 0.08 \\
\hline $\mathrm{CaO}$ & 1.69 & 1.70 & 1.33 & 0.27 & 0.28 & 0.31 & 0.20 & 0.20 & 0.38 & 0.25 & 0.39 & 0.24 & 0.36 & 0.36 & 0.37 & 0.33 & 0.32 & 0.26 & 0.34 & 0.50 & 0.42 & 0.30 & 0.47 & 0.33 \\
\hline $\mathrm{Na}_{2} \mathrm{O}$ & 4.73 & 4.67 & 4.43 & 4.10 & 3.97 & 3.95 & 4.09 & 4.11 & 3.98 & 3.96 & 3.93 & 3.90 & 4.13 & 3.78 & 3.81 & 3.89 & 3.62 & 3.92 & 3.66 & 4.16 & 3.97 & 4.20 & 4.10 & 3.96 \\
\hline $\mathrm{K}_{2} \mathrm{O}$ & 4.25 & 4.03 & 4.16 & 4.31 & 4.55 & 4.58 & 4.24 & 4.41 & 4.58 & 4.59 & 4.32 & 4.26 & 4.28 & 4.40 & 4.53 & 4.35 & 4.51 & 4.23 & 4.41 & 4.23 & 4.29 & 4.21 & 4.22 & 4.38 \\
\hline $\mathrm{P}_{2} \mathrm{O}_{5}$ & 0.12 & 0.12 & 0.09 & 0.02 & 0.02 & 0.01 & 0.01 & 0.01 & 0.01 & 0.01 & 0.02 & 0.01 & 0.01 & 0.01 & 0.01 & 0.01 & 0.01 & 0.02 & 0.01 & 0.01 & 0.01 & 0.02 & 0.01 & 0.01 \\
\hline L.O.I & 1.23 & 0.68 & 0.92 & 0.63 & 0.51 & 0.84 & 1.02 & 0.62 & 0.59 & 0.46 & 0.56 & 0.71 & 1.12 & 0.46 & 0.67 & 1.13 & 0.94 & 0.86 & 1.12 & 0.81 & 1.14 & 0.85 & 0.58 & 0.78 \\
\hline Total \% & 99.87 & 99.76 & 99.90 & 99.69 & 99.93 & 99.95 & 99.93 & 99.92 & 99.95 & 99.89 & 99.70 & 99.98 & 99.97 & 99.87 & 99.89 & 99.95 & 99.98 & 99.87 & 99.97 & 99.85 & 99.89 & 99.88 & 99.96 & 99.90 \\
\hline Q & 22.21 & 23.11 & 27.26 & 34.67 & 35.49 & 34.90 & 35.73 & 35.83 & 35.45 & 36.20 & 36.27 & 38.31 & 34.79 & 37.78 & 36.07 & 36.29 & 38.15 & 37.44 & 38.20 & 34.61 & 35.57 & 34.54 & 35.54 & 36.09 \\
\hline C & 0.00 & 0.00 & 0.01 & 0.32 & 0.17 & 0.17 & 0.41 & 0.04 & 0.00 & 0.00 & 0.30 & 0.08 & 0.28 & 0.17 & 0.08 & 0.14 & 0.23 & 0.41 & 0.28 & 0.00 & 0.19 & 0.34 & 0.11 & 0.19 \\
\hline Or & 25.11 & 23.81 & 24.55 & 25.47 & 26.89 & 27.06 & 25.06 & 26.06 & 27.06 & 27.12 & 25.53 & 25.17 & 25.29 & 26.00 & 26.77 & 25.71 & 26.65 & 25.00 & 26.06 & 25.00 & 25.35 & 24.88 & 24.94 & 25.85 \\
\hline $\mathrm{Ab}$ & 40.02 & 39.52 & 37.51 & 34.69 & 33.59 & 33.42 & 34.61 & 34.78 & 33.68 & 33.51 & 33.25 & 33.00 & 34.95 & 31.98 & 32.24 & 32.92 & 30.63 & 33.17 & 30.97 & 35.20 & 33.59 & 35.54 & 34.69 & 33.52 \\
\hline An & 4.23 & 6.13 & 4.26 & 1.22 & 1.30 & 1.51 & 0.95 & 0.95 & 0.97 & 0.54 & 1.82 & 1.16 & 1.74 & 1.73 & 1.78 & 1.59 & 1.53 & 1.23 & 1.63 & 1.99 & 2.08 & 1.49 & 2.29 & 1.48 \\
\hline $\mathrm{Di}$ & 1.59 & 0.00 & 0.77 & 0.00 & 0.00 & 0.00 & 0.00 & 0.00 & 0.43 & 0.39 & 0.00 & 0.00 & 0.00 & 0.00 & 0.00 & 0.00 & 0.00 & 0.00 & 0.00 & 0.25 & 0.00 & 0.00 & 0.00 & 0.05 \\
\hline Hy & 1.01 & 1.84 & 0.99 & 0.37 & 0.27 & 0.15 & 0.15 & 0.12 & 0.00 & 0.12 & 0.27 & 0.17 & 0.15 & 0.22 & 0.22 & 0.22 & 0.17 & 0.20 & 0.15 & 0.11 & 0.20 & 0.10 & 0.25 & 0.18 \\
\hline $\mathrm{Hm}$ & 2.97 & 2.98 & 2.58 & 2.21 & 1.51 & 1.73 & 1.86 & 1.38 & 1.48 & 1.31 & 1.48 & 1.21 & 1.46 & 1.31 & 1.83 & 1.74 & 1.48 & 1.38 & 1.39 & 1.6 & 1.61 & 1.94 & 1.38 & 1.57 \\
\hline $\mathrm{Il}$ & 0.19 & 0.19 & 0.17 & 0.08 & 0.11 & 0.08 & 0.06 & 0.06 & 0.06 & 0.13 & 0.11 & 0.04 & 0.13 & 0.09 & 0.11 & 0.09 & 0.11 & 0.06 & 0.09 & 0.11 & 0.08 & 0.08 & 0.11 & 0.09 \\
\hline $\mathrm{Ap}$ & 0.28 & 0.28 & 0.21 & 0.05 & 0.05 & 0.02 & 0.02 & 0.02 & 0.02 & 0.02 & 0.05 & 0.02 & 0.02 & 0.02 & 0.02 & 0.02 & 0.02 & 0.05 & 0.02 & 0.02 & 0.02 & 0.05 & 0.02 & 0.03 \\
\hline DI & 87.34 & 86.44 & 89.32 & 94.83 & 95.97 & 95.38 & 95.40 & 96.67 & 96.19 & 96.83 & 95.05 & 96.48 & 95.03 & 95.76 & 95.08 & 94.92 & 95.43 & 95.61 & 95.23 & 94.81 & 94.51 & 94.96 & 95.17 & 95.47 \\
\hline $\mathrm{AI}$ & 0.89 & 0.84 & 0.88 & 0.94 & 0.95 & 0.94 & 0.94 & 0.97 & 0.97 & 0.98 & 0.92 & 0.96 & 0.93 & 0.93 & 0.94 & 0.94 & 0.93 & 0.93 & 0.92 & 0.94 & 0.92 & 0.93 & 0.92 & 0.92 \\
\hline ASI & 0.90 & 0.94 & 0.94 & 1.02 & 1.01 & 1.01 & 1.03 & 1.00 & 0.97 & 0.98 & 1.02 & 1.01 & 1.02 & 1.01 & 1.00 & 1.01 & 1.02 & 1.03 & 1.02 & 0.98 & 1.02 & 1.03 & 1.01 & 1.01 \\
\hline AR & 3.70 & 3.39 & 3.85 & 5.16 & 5.41 & 5.31 & 5.23 & 5.73 & 5.65 & 6.06 & 4.88 & 5.52 & 4.92 & 5.12 & 5.24 & 5.20 & 5.26 & 5.08 & 5.10 & 4.93 & 4.85 & 4.97 & 4.77 & 5.22 \\
\hline Mg\# & 31.8 & 33.0 & 29.0 & 11.8 & 12.6 & 6.4 & 6.0 & 6.7 & 9.7 & 15.4 & 12.8 & 10.2 & 7.5 & 11.9 & 8.8 & 9.2 & 8.5 & 10.3 & 7.9 & 9.9 & 8.9 & 3.9 & 12.5 & 9.55 \\
\hline $\mathrm{Ba}$ & 494 & 671 & 514 & 51 & 134 & 117 & 69 & 90 & 80 & 39 & 58 & 95 & 63 & 49 & 3.0 & 51 & 15 & 159 & 12 & 64 & 111 & 121 & 75 & 74 \\
\hline $\mathrm{Rb}$ & 132 & 110 & 139 & 183 & 160 & 212 & 232 & 231 & 317 & 307 & 392 & 257 & 235 & 188 & 193 & 176 & 170 & 161 & 170 & 134 & 145 & 152 & 180 & 210 \\
\hline $\mathrm{Sr}$ & 188 & 218 & 177 & 17 & 41 & 30 & 17 & 16 & 20 & 18 & 21 & 37 & 19 & 12 & 12 & 15 & 9 & 51 & 8 & 19 & 34 & 48 & 35 & 24 \\
\hline $\mathrm{Nb}$ & 17 & 16 & 18 & 30 & 21 & 28 & 23 & 32 & 33 & 38 & 31 & 29 & 24 & 19 & 18 & 21 & 18 & 11 & 17 & 11 & 12 & 12 & 10 & 22 \\
\hline Y & 24 & 22 & 25 & 23 & 21 & 22 & 23 & 23 & 26 & 29 & 28 & 24 & 26 & 23 & 23 & 22 & 23 & 21 & 22 & 20 & 21 & 20 & 18 & 23 \\
\hline $\mathrm{Zr}$ & 203 & 313 & 231 & 99 & 118 & 104 & 91 & 87 & 114 & 125 & 92 & 113 & 97 & 102 & 95 & 103 & 109 & 101 & 8 & 76 & 75 & 105 & 68 & 98 \\
\hline $\mathrm{Ce}$ & 84 & 73 & 66 & 41 & 48 & 49 & 42 & 41 & 45 & 42 & 40 & 55 & 52 & 48 & 33 & 49 & 54 & 59 & 34 & 32 & 61 & 63 & 24 & 46 \\
\hline $\mathrm{Cr}$ & 10 & 8 & 12 & 25 & 10 & 20 & 5 & 5 & 3 & 10 & 1 & 8 & 2 & 4 & 2 & 3 & 2 & 6 & 1 & 6 & 5 & 9 & 2 & 6 \\
\hline $\mathrm{Ni}$ & nd & nd & 1 & nd & nd & 1 & nd & nd & nd & nd & nd & nd & nd & nd & nd & nd & nd & nd & nd & nd & nd & nd & nd & 1 \\
\hline $\mathrm{Pb}$ & 9 & 13 & 15 & 16 & 12 & 23 & 11 & 12 & 17 & 19 & 13 & 15 & 18 & 13 & 14 & 15 & 12 & 14 & 12 & 15 & 14 & 17 & 16 & 15 \\
\hline Th & 13.0 & 11.4 & 16.5 & 32.0 & 25.4 & 33.7 & 31.6 & 28.6 & 39.1 & 30.9 & 33.3 & 32.4 & 37.5 & 18.3 & 13.8 & 16.0 & 14.0 & 14.8 & 10.2 & 13.2 & 13.5 & 20.3 & 16.1 & 23.7 \\
\hline $\mathrm{U}$ & 4.07 & 5.5 & 5.2 & 9.5 & 9.8 & 10.7 & 9.6 & 8.7 & 21.3 & 8.6 & 9.5 & 7.6 & 14.3 & 5.9 & 5.2 & 6.2 & 5.8 & 4.1 & 4.8 & 3.6 & 5.7 & 7.3 & 7.9 & 8.3 \\
\hline $\mathrm{K} / \mathrm{Rb}$ & 267.5 & 304.1 & 250.6 & 195.5 & 235.9 & 179.3 & 151.7 & 158.8 & 119.9 & 124.2 & 91.49 & 137.6 & 151.1 & 193.9 & 195.2 & 205.7 & 220.6 & 218.6 & 215.2 & 261.6 & 245.3 & 229.8 & 194.5 & 186.3 \\
\hline $\mathrm{Ba} / \mathrm{Rb}$ & 3.75 & 6.10 & 3.79 & 0.28 & 0.84 & 0.55 & 0.30 & 0.39 & 0.25 & 0.13 & 0.15 & 0.37 & 0.27 & 0.26 & 0.17 & 0.29 & 0.0 & 0.99 & 0.07 & 0.47 & 0.77 & 0.79 & 0.42 & 0.39 \\
\hline $\mathrm{Rb} / \mathrm{Sr}$ & 0.70 & 0.50 & 0.81 & $\begin{array}{l}10.76 \\
10.76\end{array}$ & 3.95 & 7.09 & 13.56 & 14.23 & 15.62 & $\begin{array}{l}0.15 \\
17.52\end{array}$ & 19.12 & 6.89 & 12.38 & 15.31 & 16.76 & 11.78 & 18.86 & 3.16 & 21.00 & 7.14 & 4.26 & 3.19 & 5.09 & 11.38 \\
\hline
\end{tabular}

D.I: Differentiation Index; AI: Agpaitic Index; ASI: Alumina Saturation Index; AR: Alkalinity Ratio: $\mathrm{Mg} \#=\mathrm{Mg} \times 100 /\left(\mathrm{Mg}+\mathrm{Fe}^{2}\right)$. 
Geological, geochemical, and petrogenetic aspects

Table 2: Rare earth elements and geochemical ratios for the studied granites.

\begin{tabular}{|c|c|c|c|c|c|c|c|c|c|c|c|c|c|c|c|c|c|c|c|c|c|c|c|c|}
\hline \multirow{2}{*}{$\begin{array}{l}\text { Rock type } \\
\text { Sample No. }\end{array}$} & \multicolumn{6}{|c|}{ Monzogranite } & \multicolumn{7}{|c|}{ Syenogranite } & \multicolumn{11}{|c|}{ Alkali feldspar granite } \\
\hline & GA-1 & GA-3 & GA-6 & GA-9 & GA-12 & Av. & \begin{tabular}{|l|} 
GHS-1 \\
\end{tabular} & GHS-8 & GHS-23 & GHS-43 & O-7 & GO-11 & Av. & GH-2 & GH-3 & GH-6 & GH-7 & GH-9 & GSH-1 & GSH-4 & GSH-5 & GSH-8 & GSH-9 & Av. \\
\hline Hf & 5.33 & 5.88 & 5.56 & 5.40 & 5.10 & \begin{tabular}{|l|}
5.45 \\
\end{tabular} & 8.6 & 10.7 & 6.7 & 6.8 & 6.9 & 6.9 & 7.8 & 6.2 & 6.5 & 6.9 & 8.2 & 6.5 & 6.5 & 5.3 & 4.3 & 7.2 & 6.9 & 6.5 \\
\hline Ta & 1.02 & 1.00 & 1.05 & 1.10 & 1.20 & 1.07 & 1.2 & 1.4 & 1.2 & 1.4 & 1.1 & 1.1 & 1.2 & 2.2 & 2.4 & 2.9 & 3.4 & 2.8 & 2.6 & 1.5 & 1.3 & 2.7 & 2.4 & 2.4 \\
\hline La & 27.98 & 31.40 & 31.69 & 26.39 & 31.10 & 29.71 & 37.9 & 47.9 & 38.7 & 35.80 & 34.1 & 35.10 & 38.25 & 35.3 & 30.1 & 28.3 & 19.1 & 34.5 & 29.2 & 25.4 & 24.4 & 29.8 & 29.5 & 28.56 \\
\hline $\mathrm{Ce}$ & 58.00 & 65.24 & 65.50 & 51.30 & 50.50 & 58.11 & 75 & 70.9 & 67.9 & 64.90 & 67.3 & 72.00 & 69.67 & 45.5 & 46.9 & 40.9 & 37.9 & 52.3 & 45.2 & 51 & 57.1 & 57.7 & 59.1 & 49.36 \\
\hline $\mathrm{Pr}$ & 7.10 & 6.98 & 7.20 & 8.40 & 7.60 & 7.46 & 7.16 & 8.41 & 6.1 & 6.12 & 6.4 & 6.70 & 6.82 & 4.9 & 3.52 & 2.85 & 2.17 & 4.15 & 3.5 & 4.84 & 5.54 & 4.5 & 5.6 & 4.16 \\
\hline Nd & 24.19 & 25.23 & 25.70 & 21.10 & 20.20 & 23.28 & 31.2 & 37.2 & 25.8 & 25.10 & 26.6 & 29.10 & 29.17 & 14.2 & 8.9 & 9.8 & 4.4 & 11.8 & 9.3 & 17.1 & 21.3 & 16.9 & 23.8 & 13.75 \\
\hline Sm & 5.05 & 3.81 & 4.67 & 6.50 & 7.10 & 5.43 & 6.1 & 6.91 & 5.1 & 4.98 & 5.31 & 5.49 & 5.65 & 1.8 & 1.24 & 1.49 & 0.48 & 1.51 & 1.28 & 3.21 & 3.95 & 3.41 & 4.78 & 2.32 \\
\hline Eu & 1.13 & 0.90 & 1.01 & 1.10 & 1.12 & 1.0 & 1. & 1.05 & 0.9 & 0.84 & 0.89 & 1.26 & 1.04 & 0.12 & 0.1 & 0.12 & 0.03 & 0.15 & 0.12 & 0.16 & 0.36 & 0.4 & 0.65 & 0.22 \\
\hline Gd & 3.48 & 3.87 & 3.70 & 5.20 & 5.90 & 4.43 & 5. & 5.1 & 4.3 & 4.5 & 4.8 & 4.81 & 4.8 & 1.27 & 0.94 & 1.05 & 0.42 & 1.06 & 0.96 & 3.1 & 2.95 & 3.4 & 4.2 & 1.94 \\
\hline Tb & 0.57 & 0.49 & 0.46 & 0.60 & 0.58 & 0.54 & 07 & 0.7 & 0.6 & 062 & 0.61 & 0.70 & 0.66 & 0.25 & 0.18 & 0.12 & 01 & 0.19 & 0.17 & 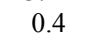 & 0.49 & 0.51 & 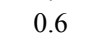 & 0.30 \\
\hline Dy & 2.73 & 3.55 & 2.82 & 3.50 & 3.90 & & & 3.95 & & & & & & 1.57 & 1.26 & 1.3 & 0.86 & 1.32 & 1.25 & 2.79 & 65 & 3.1 & & 1.94 \\
\hline Ho & 0.53 & 0.67 & 0.52 & 0.54 & 0.64 & 0.5 & 0.81 & 0.84 & 0.65 & 0.6 & 0.71 & 0.70 & 0.73 & 0.54 & 0.32 & 0.23 & 0.27 & 0.31 & 0.3 & 0.59 & .53 & 0.59 & 0.6 & 0.43 \\
\hline Er & 1.44 & 2.39 & 1.45 & 2.10 & 2.20 & 1. & & 2.16 & & & 20 & & 2.1 & 1.56 & 1.28 & & 1.27 & 1.27 & 1.26 & 2 & 47 & 2.2 & 1.79 & 1.50 \\
\hline Tm & 0.28 & 0.32 & 0.19 & 0.31 & 0.30 & 0.28 & 0.36 & 0.4 & 0.27 & 0.31 & 0.32 & 0.31 & 0.33 & 0.25 & 0.26 & 0.2 & 0.3 & 0.25 & 0.24 & 0.3 & 0.18 & 0.34 & 0.24 & 0.26 \\
\hline Yb & 1.44 & 2.38 & 1.62 & 1.70 & 1.90 & 1.81 & & & & 2. & & & 2.2 & 1.67 & 2.39 & 1.6 & 2.9 & 2.45 & & & & & & 2.14 \\
\hline Lu & 0.25 & 0.32 & 0.27 & 0.30 & 0 & 0 & & & & 0. & 0.38 & & 0.37 & 0.27 & 0.43 & 0.26 & 0.5 & 0.44 & 0.44 & 0.35 & 0.26 & 0.41 & 0.29 & 0.37 \\
\hline$\Sigma$ REE & 134.2 & 147.5 & 146.8 & 129.0 & 133.3 & 138.2 & 175.6 & 188.3 & 158.0 & 151.9 & 155.4 & 164.6 & 165.6 & 109.2 & 97.8 & 89.2 & 70.8 & 111.7 & 95.6 & 113.4 & 122.7 & 125.9 & 136.2 & 107.02 \\
\hline $\mathbf{L a}_{N} / \mathbf{L} \mathbf{u}_{N}$ & 11.63 & 10.19 & 12.18 & 9.13 & 11.53 & 10.93 & & & & 054 & 9.32 & & 10. & 13.6 & 7.27 & 11 & 3. & & 6. & 7.5 & 9.79 & & & 8.63 \\
\hline $\mathbf{L a}_{\mathbf{N}} / \mathbf{Y} \mathbf{b}_{\mathbf{N}}$ & 13.10 & 8.89 & 13.2 & 10.5 & 11 & 11.34 & 10.2 & 13.5 & 13.1 & 11 & 10.9 & 11.3 & 11.65 & 14.3 & 8.49 & 11.8 & 4.43 & 9.49 & 8.34 & 8.15 & 10.7 & 7.73 & 11.1 & 9.44 \\
\hline $\mathbf{L a}_{\mathbf{N}} / \mathbf{S} \mathbf{m}_{\mathbf{N}}$ & 3.49 & 5.18 & 4.27 & 2.55 & 2.76 & 3.65 & 3.91 & 4.36 & 4.77 & 4.52 & 4.0 & 4.0 & 4.27 & 12.3 & 15.3 & 12 & 25 & 14.4 & 14.4 & 4.98 & 3.89 & 5.5 & 3.88 & 11.18 \\
\hline G & 1.72 & 1.50 & 1.7 & 2.15 & 2.62 & 1.94 & 1.61 & 1.81 & 1.57 & 1.45 & 1.57 & 1.66 & 1.6 & 0.58 & 0.27 & 0.5 & 0.09 & 0.3 & 0.27 & 1.1 & 1.41 & 1.03 & 1.8 & 0.74 \\
\hline $\mathbf{G d}_{\mathbf{N}} / \mathbf{Y l}$ & 1.93 & 1.31 & 1.84 & 2.46 & 2.51 & 2.01 & 1.7 & 1.72 & 1.74 & 1.66 & 1.83 & 1.85 & 1.75 & 0.61 & 0.32 & 0.52 & 0.17 & 0.35 & 0.33 & 1.19 & 1.55 & 1.06 & 1.88 & 0.80 \\
\hline $\mathbf{E u} / \mathbf{E u} \mathbf{u}^{*}$ & 0.82 & 0.72 & 0.74 & 0.58 & 0.53 & 0.68 & 0.7 & 0.54 & 0.59 & 0.54 & 0.54 & 0.75 & 0.61 & 0.24 & 0.28 & 0.29 & 0.2 & 0.36 & 0.33 & 0.15 & 0.32 & 0.36 & 0.44 & 0.30 \\
\hline
\end{tabular}




\section{El Afandy, et al.}

REEs data for the studied granitic rocks (Table 2) are shown in chondrite-normalized diagrams (Fig. 7). The average of total REE contents of the studied monzogranites, syenogranites and alkali feldspar granites are (138.18, 165.24 and 107.24 respectively). In general, the REE patterns of the different granitic rock types of the study area are enriched in LREE relative to the HREE; with $\left(\mathrm{La}_{\mathrm{n}} / \mathrm{Yb}_{\mathrm{n}}\right)$ values vary from 8.89 and 13.19 for the monzogranites, from 10.18 to 13.45 for the syenogranites and from 4.43 to 14.25 for the alkali feldspar granites. Fractionation of zircon, apatite and allanite could cause a reduction in LREE abundance (Hanson, 1978).

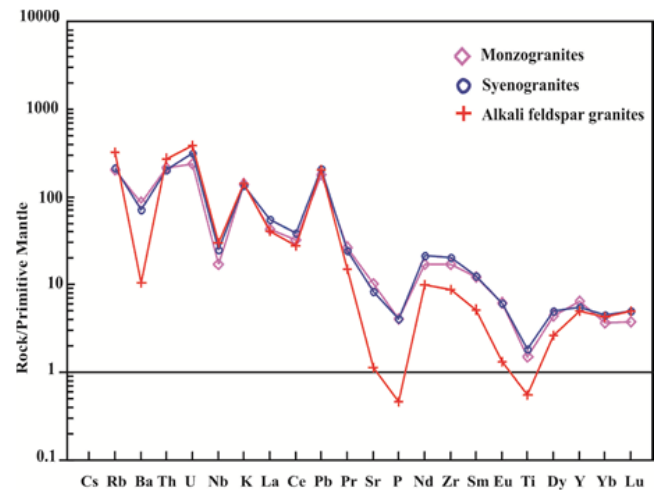

Fig.6: Multi-element distribution diagrams for the studied granites. Normalization values after Sun and McDonough (1989).

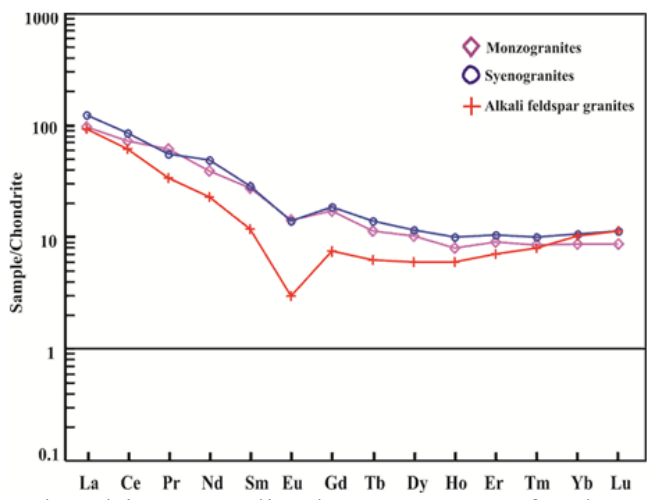

Fig. 7: Chondrite normalized REE patterns for the studied granites. Normalization values after Sun and McDonugh (1989).

In the highly differentiated granites, the $\mathrm{Rb}$ content tends to enrich relative to $\mathrm{K}$ (Taylor et al., 1956). Thus, it is quite possible to expect that the $\mathrm{K} / \mathrm{Rb}$ ratio would be low in strongly differentiated granites. Igneous rocks derived from upper mantle have $\mathrm{K} / \mathrm{Rb}$ ratio ranges from 700 to 1500 (Heier, 1973), while it ranges from 150 to 350 in granitic crustal rocks (Taylor, 1965). In the present study, $\mathrm{K} / \mathrm{Rb}$ ratio decreases from the monzogranites ( 273 on average) through the syenogranite (250 on average) to the alkali feldspar granite (186 on average) (Table. 1). The $\mathrm{Rb} / \mathrm{Sr}$ ratio increases with magmatic differentiation due to $\mathrm{Sr}$ depletion in liquid magmas as a result of crystallization of feldspars In the present study, monzogranites and syenogranites have lower $\mathrm{Rb} / \mathrm{Sr}$ ratios with (average $=0.62$ and 0.81 respectively) than alkali feldspar granites (average $=11.38)($ Table 1$)$, this may be related to the decrease the plagioclase amount in the latter. Gast (1965) and Heier (1973) have shown that, high $\mathrm{K} / \mathrm{Rb}$ ratios and low $\mathrm{Rb} / \mathrm{Sr}$ ratios are characteristic features for rocks that derived from lower crust or upper mantle. On the contrary, the younger granites have low $\mathrm{K} / \mathrm{Rb}$ ratio, and high $\mathrm{Rb} / \mathrm{Sr}$ ratio indicating their formations from the granitic crustal rocks.

The LREE of monzogranites and syenogranites are moderately fractionated $\left(\mathrm{La}_{\mathrm{n}} / \mathrm{Sm}_{\mathrm{n}}=2.55-5.18\right.$ and $3.91-4.77$, respectively) and moderately to highly fractionated in alkali feldspar granite $\left(\left(\mathrm{La}_{\mathrm{n}} / \mathrm{Sm}_{\mathrm{n}}\right.\right.$ $=3.88-25.03)$. The monzogranite exhibits moderately negative $\mathrm{Eu}$ anomalies $\left(\mathrm{Eu} / \mathrm{Eu}^{*}=0.53-0.82\right)$ indicating plagioclase fractionation. The REE pattern of the syenogranite is closely similar to the pattern of the monzogranite but with higher abundances in all REEs. It displays moderately negative $\mathrm{Eu}$ anomalies $\left(\mathrm{Eu} / \mathrm{Eu}^{*}=0.54-0.75\right)$. The alkali feldspar granite has strongly negative $\mathrm{Eu}$ anomalies $\left(\mathrm{Eu} / \mathrm{Eu}^{*}=0.15-0.44\right)$. The extremely negative Eu anomaly values in the alkali feldspar granite can be attributed to the removal of plagioclases from the source magma by fractional crystallization (Lee et al., 2013).

\section{Magma type}

The chemical nature and behavior of the magma during its evolution for the studied granitic rocks can be identified using the following.

On the $\mathrm{SiO}_{2}(\mathrm{wt} \%)$ vs. $\log \left(\mathrm{K}_{2} \mathrm{O} / \mathrm{MgO}\right)$ diagram (Rogers and Greenberg, 1981), the studied monzogranites and syenogranites plot in the overlap zone between calc-alkaline and alkali granite fields (Fig. 8), while the alkali feldspar granites plot within the field of the alkali granites. The studied granitic rocks are straddling the boundary line between metaluminous to peraluminous fields on the A/NK vs. A/CNK variation diagram of Manair and Piccoli (1989), suggesting metaluminous to weakly peraluminous character(Fig. 9). 
Geological, geochemical, and petrogenetic aspects

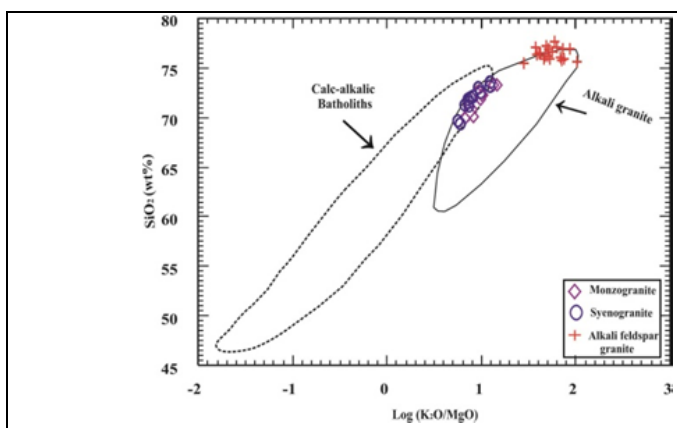

Fig. 8: $\mathrm{SiO}_{2}$ (wt. \%) vs. $\log \mathrm{K}_{2} \mathrm{O} / \mathrm{Na}_{2} \mathrm{O}$ for the studied granites, diagram after Rogers and Greenberg, (1981).

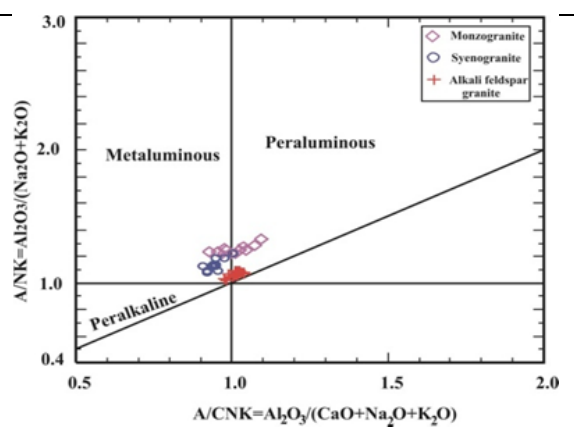

Fig. 9: Molar A/NK vs. A/CNK for the study granites, diagram after Maniar and Piccoli, (1989).

In terms of Agpaitic index (AI) versus $\mathrm{SiO}_{2} \mathrm{wt} \%$ diagram of Lie'geois and Black (1987), the studied monzogranites plot in the field of calc-alkaline metaluminous domain (Fig. 10), whereas the studied syenogranites and alkali feldspar granites plot in the alkaline metaluminous domain except three syenogranite samples plot in the calc-alkaline metaluminous domain.

Based on the $\left(\mathrm{Na}_{2} \mathrm{O}+\mathrm{K}_{2} \mathrm{O}-\mathrm{CaO}\right)$ versus $\mathrm{SiO}_{2} \mathrm{wt} \%$ binary diagram (Figs. 11) after Frost et al. (2001), the investigated granites are mainly plot in the calc-alkalic to alkalic-calcic fields. Some granitoid's classification schemes enlarged the granitic spectrum between two contrasted endmembers, mantle-derived (M-type) and crustal-derived (C-type) varities. The M-type granite is thought to arise from the mantle, specifically in island arc settings (White \& Chappell, 1983). The Ctype granites are defined as crustal/ charnockitic granitoids (Kilpatrick \& Ellis, 1992). The studied granitic rocks plot consistently in the field of C-type granites (Fig. 12) indicating that they were derived from crustal sialic materials. The $\mathrm{SiO}_{2} \mathrm{wt} \%$ versus $\mathrm{Rb} / \mathrm{Zr}$ variation diagram (Harris et al., 1986) discriminates between I-type and S-type granites. The studied granite samples plot in the field of I-type granites (Fig. 13).

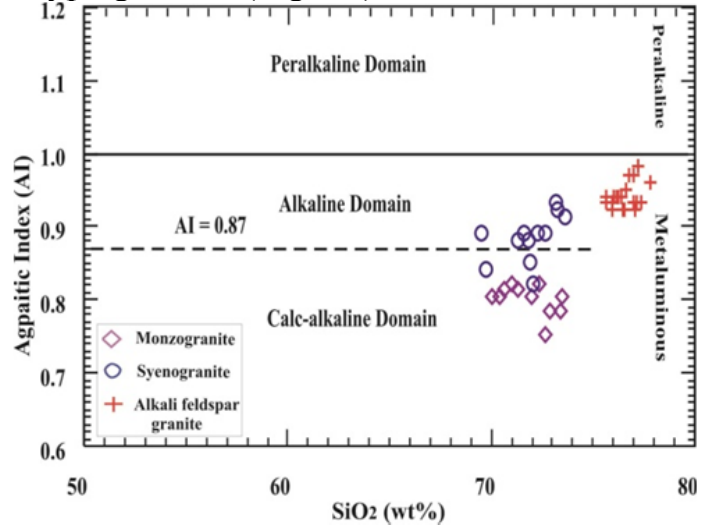

Fig.10: Agpaitic index (AI) vs. $\mathrm{SiO}_{2}(\mathrm{wt} \%$ ) diagram for the studied granites. The dashed line with $\mathrm{AI}=0.87$ separates alkaline and calc-alkaline granitoid series.

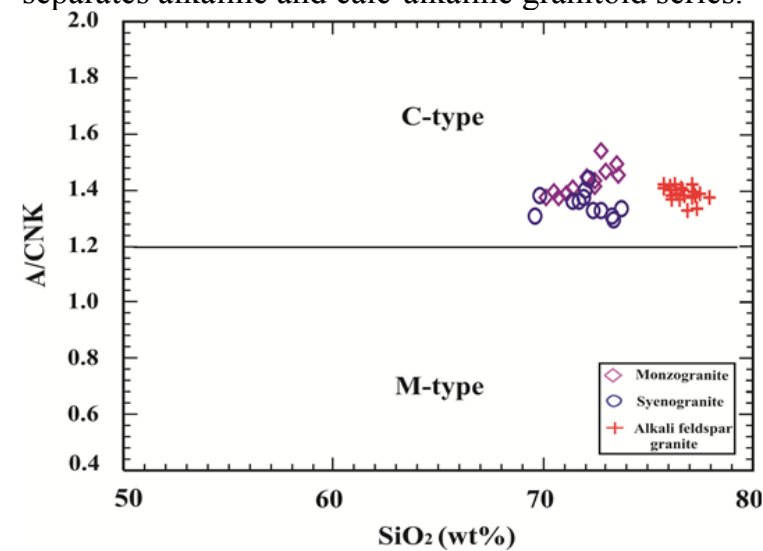

Fig. 12: $\mathrm{SiO}_{2}$ (wt. \%) vs. $\mathrm{Al}_{2} \mathrm{O}_{3} /\left(\mathrm{CaO}+\mathrm{Na}_{2} \mathrm{O}+\mathrm{K}_{2} \mathrm{O}\right)$ for the studied granites (diagram after Didier et al. 1982)

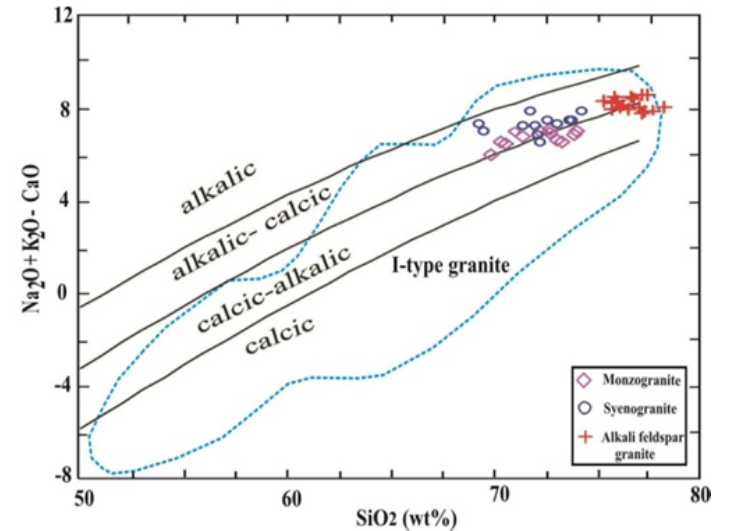

Fig. 11: Discrimination diagrams after Frost et al., (2001) for the studied granites.

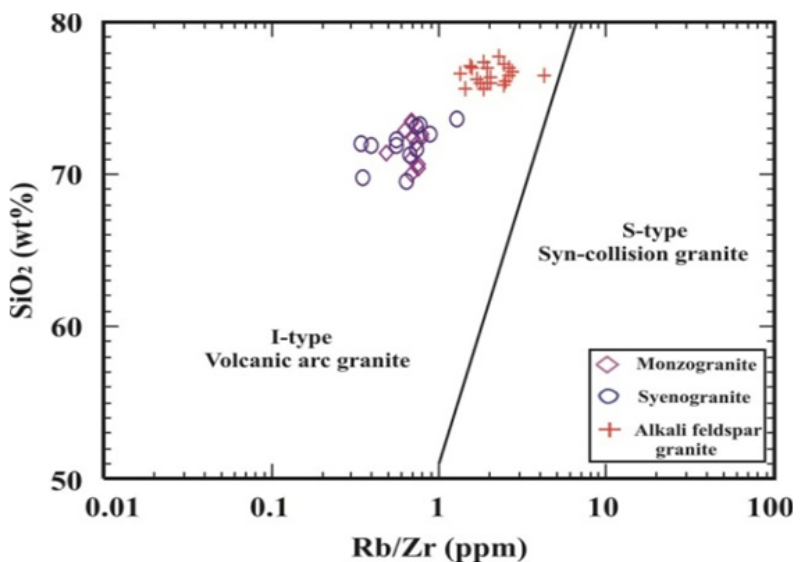

Fig. 13: $\mathrm{Rb} / \mathrm{Zr}$ vs. $\mathrm{SiO}_{2}(\mathrm{wt} \%$ ) for the studied granites. (diagram after Harries et al., 1986. 


\section{El Afandy, et al.}

On the $\mathrm{FeOt} / \mathrm{MgO}$ and $\left(\mathrm{K}_{2} \mathrm{O}+\mathrm{Na}_{2} \mathrm{O}\right) / \mathrm{CaO}$ versus $\mathrm{Zr}+\mathrm{Nb}+\mathrm{Ce}+\mathrm{Y}$ diagrams (Figs. $14 \mathrm{a} \& \mathrm{~b}$ ) (Whalen et al., 1987), the most of the studied granite samples scatter within the field of fractionated granites (FG) and beyond it. Thus, the concerned granites show significant compositional overlapping between the highly fractionated I- type and A-type granites. This overlap is observed when comparing the average chemical compositions of the investigated granites with those of the felsic I- and A-type granites from different environments. From these relations, it is concluded that the studied granites are metaluminous to slightly peraluminous and highly fractionated I-type granites.
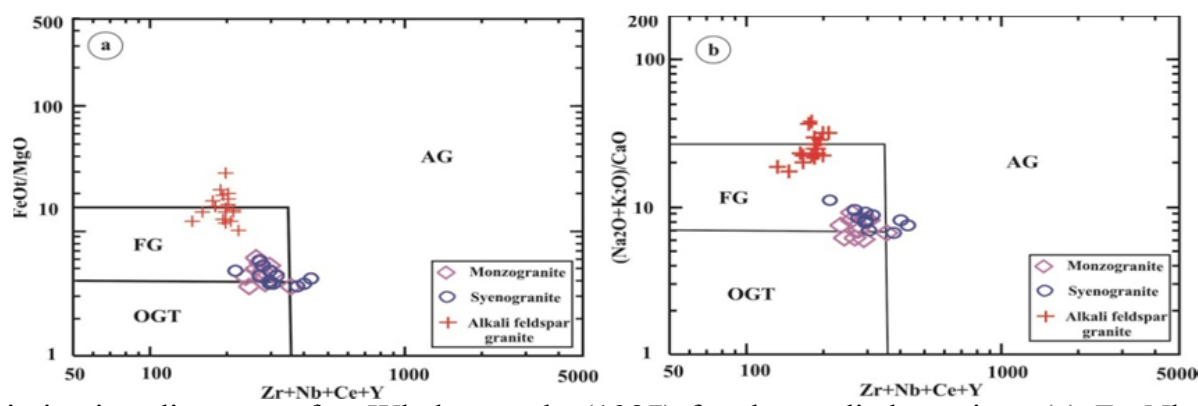

Fig.14: Discrimination diagrams after Whalen et al., (1987) for the studied granites; (a) $\mathrm{Zr}+\mathrm{Nb}+\mathrm{Ce}+\mathrm{Y}$ versus $\mathrm{FeOt} / \mathrm{MgO}$ and (b) $\mathrm{Zr}+\mathrm{Nb}+\mathrm{Ce}+\mathrm{Y}$ vs. $\left(\mathrm{K}_{2} \mathrm{O}+\mathrm{Na}_{2} \mathrm{O}\right) / \mathrm{CaO}$. FG: Fractionated felsic granites; OGT: Orogenic granite types (unfractionated M-, I- and S-types); AG: A-type alkaline granite.

\section{Tectonic setting}

The R1-R2 binary diagram (Fig. 15) of Batchelor and Bowden (1985) shows that, the monzogranite and syenogranite samples fall in the field of syn-collision granites, while the alkali feldspar granites fall in the post-orogenic field.

Pearce et al. (1984) stated that volcanic arc granites (VAG) can vary in setting from oceanic to continental and in composition from tholeiitic through calc- alkaline to shoshonitic. They defined collisional granites as syn- or post-tectonic granites associated with continent continent collision, or syn-tectonic granites associated with continent arc. $\mathrm{On} \mathrm{Rb}(\mathrm{ppm})$ versus $\mathrm{Y}+\mathrm{Nb}(\mathrm{ppm})$ discrimination diagram of Pearce et al., (1984) (Fig. 16), the monzogranites and syenogranites fall in the field of volcanic arc granites (VAG). Alkali feldspar granites plot in the upper part in the field of volcanic arc granites and very close to the field of within plate granites (WPG); the studied granites plot within the field of post-collision granites (Pearce, 1996). The post-tectonic granites most commonly plot in the same region as volcanic arc granites, belong to calc-alkalic suites, metaluminous to slightly peraluminous, and exhibit most of the characteristics of I-type granites (Pearce et al., 1984).

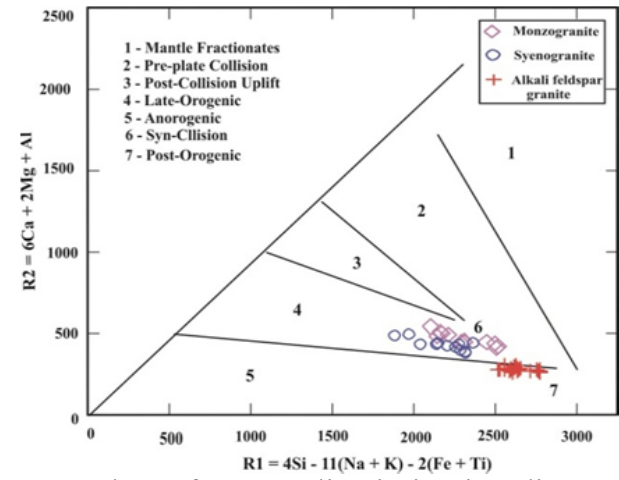

Fig. 15: Plots of R1-R2 discrimination diagram for the studied granites (after Batchelor and Bowden; 1985).

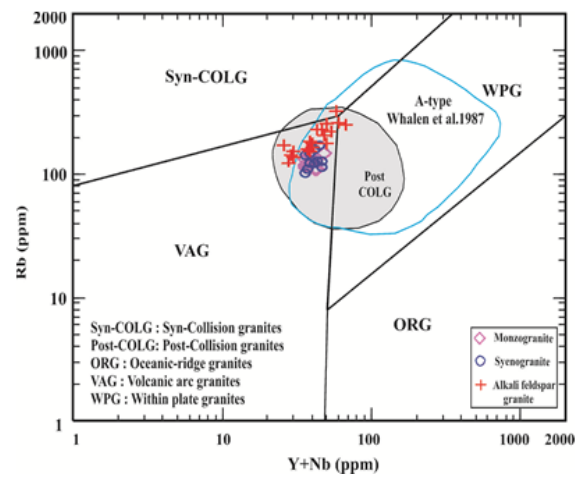

Fig. 16: Plots of $\mathrm{Rb}(\mathrm{ppm}) \mathrm{vs.} \mathrm{Y}+\mathrm{Nb}$ for the studied granites on the Tectonic discrimination diagrams (after Pearce et al., 1984). The post collision granite field after Pearce, 1996).

\section{PETROGENESIS}

\section{Source rock}

Several source rocks have been assumed to elucidate the generation of granitic magmas by partial melting mechanism. Partial melting under variable conditions of various source rocks output granitic melts of different composition. The source rocks from which the granitoids were generated can be deduced through the following relationships:- 
Based on the variations of the $\mathrm{Al}_{2} \mathrm{O}_{3}, \mathrm{CaO}, \mathrm{MgO}, \mathrm{FeOt}, \mathrm{K}_{2} \mathrm{O}$ and $\mathrm{Na}_{2} \mathrm{O}$ contents, the ternary discrimination diagram of Laurent et al (2014), shows that the studied granites are derived from tonalites (intermediate magmas ) or crustal sources (Fig. 17).

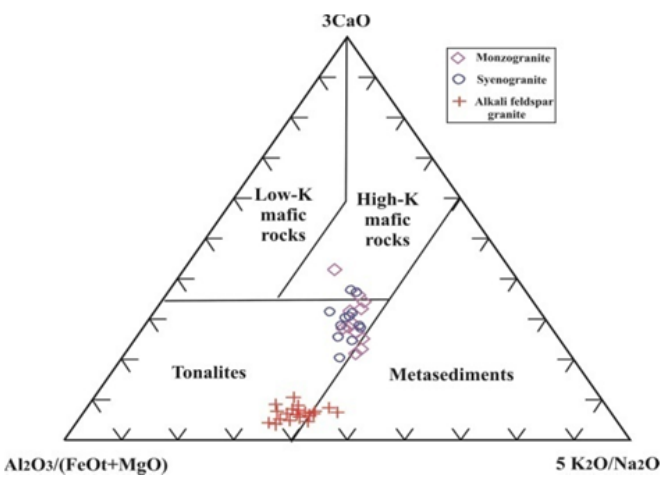

Fig. 17: $\mathrm{Al}_{2} \mathrm{O}_{3} /(\mathrm{FeOt}+\mathrm{MgO})-3 \mathrm{CaO}-$ $5 \mathrm{~K}_{2} \mathrm{O} / \mathrm{Na}_{2} \mathrm{O}$ ternary diagram for the studied granites (after Laurent et al., 2014)

\section{Magma conditions}

The CIPW normative compositions are more useful tools in giving some information about the P-T conditions of granitic magma crystallization. On the Ab-Q-Or ternary diagram (Fig. 18) of Tuttle and Bown (1958), the study granites were generated in the 0.5 - $10 \mathrm{kbar}$ (Luth et al., 1964). The investigated granites lie in the granite field and are clustered around the isobaric univariant curve, and at the minimum water vapor pressure ( 1 kbar; James and Hamilton, 1969) suggesting crystallization at relatively shallower depth and the crystal-liquid equilibrium was the dominant mechanism involved in the genesis of these granites (James and Hamilton, 1969). Using the thermal lines (James and Hamilton, 1969) on the Ab-Q-Or ternary diagram, the study granitoids have temperatures of about $650^{\circ} \mathrm{C}-670^{\circ} \mathrm{C}$.

Fig. 18: Normative Ab-Q-Or diagram for the study granites. Solid lines represent 0.5 and 10 kbar pH2O (after Luth et al., 1964). Dotted lines represent the trace of isobaric minimum of eutectic points at intermediate water vapour pressure

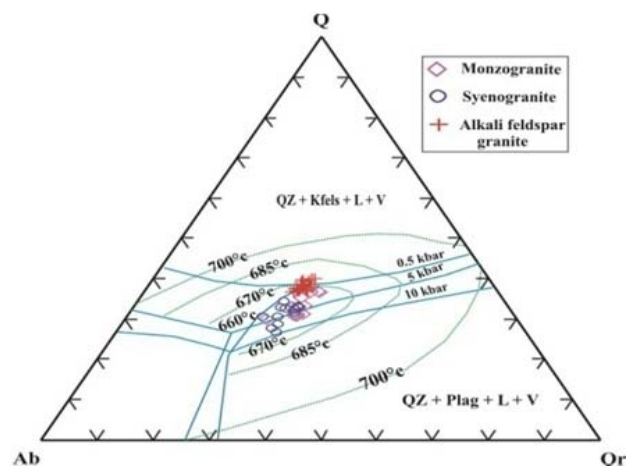

On the normative Ab-An-Or ternary diagram (Fig. 19) illustrates that the analyzed monzogranites and syenogranites were generated at moderate/high water pressure $\left(<8 \mathrm{~kb} \mathrm{P}_{\mathrm{H} 2 \mathrm{O}}>5 \mathrm{~kb} \mathrm{P}_{\mathrm{H} 2 \mathrm{O}}\right.$ and $<7 \mathrm{~kb}$ $\mathrm{P}_{\mathrm{H} 2 \mathrm{O}}>3 \mathrm{~kb} \mathrm{P}_{\mathrm{H} 2 \mathrm{O}}$; Winkler et al., 1975) suggesting crystallization at moderate depth. The plotting of the alkali feldspar granites suggests crystallization at low water vapor pressure about $1 \mathrm{kbar}$. According to James and Hamilton, the estimated temperatures are about $660-685^{\circ} \mathrm{C}$ for monzogranites and syenogranites, and about $660^{\circ} \mathrm{C}$ for alkali feldspar granites. Most of the studied granites have compositions close to the minimum melting point at low to moderate $\mathrm{P}_{\mathrm{H} 2 \mathrm{O}}$ indicating that these granites have been originated by partial melting within the crust (Presenall and Bateman, 1973). Such conditions may be generated within a thickened crust and may be due to its relaxation at the end of plate collision (late tectonic) or an I-type magma tectonic setting.

A binary relation between $\mathrm{Rb}$ (ppm) and $\mathrm{Sr}$ (ppm) (Fig. 20) suggested by Condie (1973) to estimate the crustal thickness. The monzogranites and syenogranites have been generated at greater depth $>30$ $\mathrm{km}$ of the lower crust, while the alkali feldspar granites have been emplaced at relatively shallow to moderate depths between 20 and $30 \mathrm{~km}$.

\section{Fractionation processes}

The overall trends of trace elements (Fig. 5) and the fractionated nature of the REE $\left[(\mathrm{La} / \mathrm{Lu})_{\mathrm{n}}=\right.$ 3.67-14.21] suggest fractional crystallization was played the major role during the evolution of the study granites. This is supported by the decrease in $\mathrm{FeO}^{\mathrm{t}}, \mathrm{MgO}, \mathrm{CaO}, \mathrm{TiO}_{2}$, and $\mathrm{P}_{2} \mathrm{O}_{5}$ with increasing $\mathrm{SiO}_{2}$ coupled with increasing $\mathrm{Rb}, \mathrm{Y}$, Th and $\mathrm{Nb}$ are consistent with fractional crystallization. Also, the 


\section{El Afandy, et al.}

striking depletions in $\mathrm{P}, \mathrm{Ti}, \mathrm{Ba}, \mathrm{Sr}, \mathrm{Nb}$, and $\mathrm{Eu}$ in the various types of granites on the spider diagrams (Fig. 7) support an interpretation favoring fractional crystallization process. Negative Nb-Ti anomalies are considered to be related to fractionation of Ti-bearing phases (ilmenite and titanite) and negative $\mathrm{P}$ anomalies are resulted from apatite separation. Strong Eu depletion requires extensive fractionation of plagioclase and/or K-feldspar. Fractionation of plagioclase implies negative $\mathrm{Sr}-\mathrm{Eu}$ anomalies, and that of $\mathrm{K}$-feldspar produces negative $\mathrm{Eu}-\mathrm{Ba}$ anomalies.

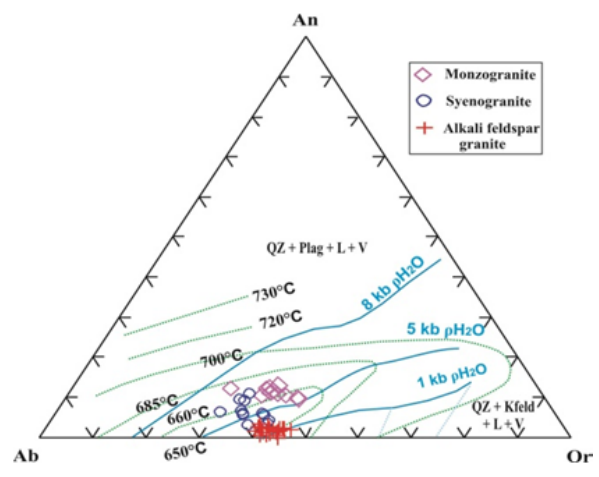

Fig. 19: Normative Ab-An-Or diagram for the studied granites. The solid isobaric blue lines represent the two feldspar boundary curves for the quartz-saturated ternary feldspar system at 1 and $5 \mathrm{kbar}, 8 \mathrm{kbar} \mathrm{P}_{\mathrm{H} 2 \mathrm{O}}$. Dotted red lines represent temperature of crystallization (after James and Hamilton, 1969).

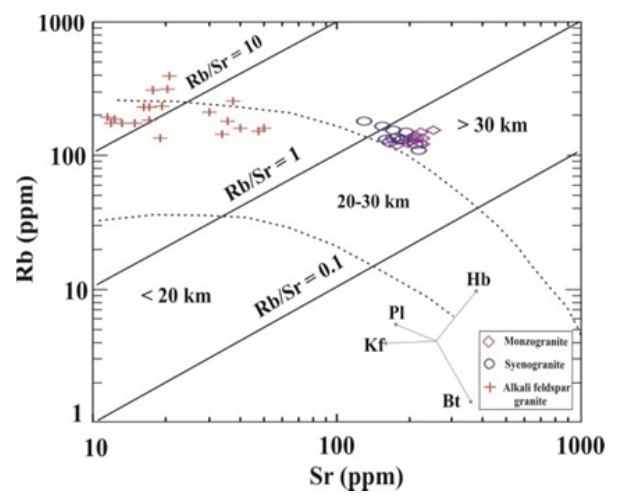

Fig. 20: Plotting of the studied granites on Sr (ppm) vs. $\mathrm{Rb}(\mathrm{ppm})$ binary diagram. The dashed lines refer to the crustal thickness (after Condie 1973).

\section{SUMMARY AND CONCLUSIONS}

The field, petrography and geochemical investigation of the studied younger granites these granites distinguished to monzogranite, syenogranite and alkali feldspar granite.

Monzogranites are mainly exposed along W. Um Sidra at G. Abu Mesaid in the eastern parts of the studied area, forming low to moderate topographic reliefs. These rocks intrude the Dokhan volcanic with sharp contacts and send several offshoots into them. This granite type is composed of potashfeldspar (orthoclase and microcline perthite), plagioclases $\left(\mathrm{An}_{14-29}\right)$, quartz, biotite and hornblende as essential minerals. Opaque minerals, sphene, allanite, zircon and apatite are the accessory minerals while epidote and chlorite are secondary minerals. Syenogranites are exposed at G. El Shagola, G. Abu Harba, G Abu Mesaid, and some parts along W. Um Sidra intruding the the metavolcanics and Dokhan volcanics with sharp contacts and contains enclaves of them. They are mainly composed of potash feldspar (orthoclase and microcline perthites), quartz, plagioclases $\left(\mathrm{An}_{8-20}\right)$ and biotite as essential minerals. The accessory minerals include zircon, sphene, muscovite, apatite and iron oxides, while epidote and chlorite are secondary minerals. Alkali feldspar granites are exposed at G. El Shaylah, G. Urf El Eir, G. El Hurus, and the western exposures of G. Abu Harba intruding the metavolcanics and Dokhan volcanics with sharp and irregular contacts. These rocks composed of potash feldspar (orthoclase and microcline perthites), quartz, plagioclases $\left(\mathrm{An}_{5-15}\right)$ and few biotite as essentially minerals. The main accessory minerals are zircon, sphene, apatite, allanite and iron oxides, while Muscovite, chlorite, sericite and epidote are secondary minerals.

The studied monzogranites and syenogranites are originated from calc-alkaline magma whereas the alkali feldspar granites have alkaline characters. The $\mathrm{K} / \mathrm{Rb}, \mathrm{Ba} / \mathrm{Rb}$ and $\mathrm{Rb} / \mathrm{Sr}$ ratios for the studied granites clear that they originated from highly differentiated magma. These granites were generated in possible extensional environment and in a post-collision granites environment. The post-collision granites belong to calc-alkalic suites, are metaluminous to slightly peraluminous, and exhibit most of the characteristics of highly fractionated I-type granites derived from tonalites (intermediate magmas) or crustal sources.

The monzogranites and syenogranites were generated at moderate/high water pressure in the $3-8$ kbar and temperatures of about $660^{\circ} \mathrm{C}-685^{\circ} \mathrm{C}$. The alkali feldspar granites crystallized more or less at the same temperature but at lower water vapor pressure about $1 \mathrm{kbar}$, suggesting crystallization at relatively shallower depth. The crystal-liquid equilibrium was the dominant mechanism involved in the genesis of these granites. The monzogranites and syenogranites have been generated at greater 
Geological, geochemical, and petrogenetic aspects

depth $>30 \mathrm{~km}$ of the lower crust, while the alkali feldspar granites have been emplaced at relatively shallow to moderate depths between 20 and $30 \mathrm{~km}$.

The overall decreasing trends in $\mathrm{FeO}^{\mathrm{t}}, \mathrm{MgO}, \mathrm{CaO}, \mathrm{TiO}_{2}$, and $\mathrm{P}_{2} \mathrm{O}_{5}$ with increasing $\mathrm{SiO}_{2}$ coupled with increasing $\mathrm{Rb}, \mathrm{Y}, \mathrm{Th}$ and $\mathrm{Nb}$ and the fractionated nature of the REE $\left[(\mathrm{La} / \mathrm{Lu})_{\mathrm{n}}=3.67-14.21\right]$ suggesting that the fractional crystallization was played the major role during the evolution of the studied granites. Also, the striking depletions in $\mathrm{P}, \mathrm{Ti}, \mathrm{Ba}, \mathrm{Sr}, \mathrm{Nb}$, and $\mathrm{Eu}$ in the various types of granites on the spider diagrams support the interpretation of fractional crystallization process. Negative $\mathrm{Nb}$-Ti anomalies are considered to be related to fractionation of Ti-bearing phases (ilmenite and titanite) and negative $\mathrm{P}$ anomalies are resulted from apatite separation. Strong Eu depletion requires extensive fractionation of plagioclase and/or K-feldspar. Fractionation of plagioclase implies negative $\mathrm{Sr}-\mathrm{Eu}$ anomalies, and that of $\mathrm{K}$-feldspar produces negative $\mathrm{Eu}-\mathrm{Ba}$ anomalies.

\section{REFERENCES}

Ali, B. H., (2015): SHRIMP U-Pb zircon geochronology: evidence for emplacement time of some granitoids north Eastern Desert, Egypt. Arab. J. Geosci. 8, 5465-5474.

Ali, B. H., Wilde, S. A., and Gabr, M. M. A., (2009a): Granitoid evolution in Sinai, Egypt, based on precise SHRIMP U-Pb zircon geochronology. Gondwana Res. 15, 38-48.

Ali, K. A., Moghazi, A. K. M., Maurice, A. E., Omar, S. A., Wang, Q., Wilde, S. A., Moussa, E. M., Manton, W. I., and Stern, R. J., (2012): Composition, age, and origin of the $\sim 620$ Ma Humr Akarim and Humrat Mukbid A-type granites: no evidence for pre-Neoproterozoic basement in the Eastern Desert, Egypt. Int. J. Earth Sci. 101, 1705-1722.

Ali, K. A., Stern, R. J., Manton, W. I., Kimura, J.I., and Khamees, H. A., (2009b): Geochemistry, Nd isotopes and U-Pb SHRIMP zircon dating of Neoproterozoic volcanic rocks from the Central Eastern Desert of Egypt: New insights into the $\sim 750$ Ma crust-mforming event. Precamb. Res. 171, $1-22$.

Ali, K. A., Wilde, S. A., Stern, R. J., Moghazi, A. K. M., and Ameen, S. M., (2013): Hf isotopic composition of single zircons from Neoproterozoic arc volcanics and post-collision granites, Eastern Desert of Egypt: Implications for crustal growth and recycling in the Arabian-Nubian Shield. Precamb. Res. 239, 42-55.

Ali, K. A., Zoheir, B.A., Stern, R.J., Andresen, A., Whitehouse, M.J. and Bishara, W.W., (2016): LuHf and O isotopic compositions on single zircons from the North Eastern Desert of Egypt, Arabian-Nubian Shield: Implications for crustal evolution. Gondwana Research 32, 181-192.

Asran, A.M., Emam, A., and El-Fakharani, A., (2017): Geology, structure, geochemistry and ASTERbased mapping of Neoproterozoic Gebel El-Delihimmi granites, Central Eastern Desert of Egypt. Lithos 282, 358-372.

Azer, M. K., (2013): Late Ediacaran (605-580 Ma) post-collisional alkaline magmatism in the Arabian-Nubian Shield: a case study of Serbal ring-shaped intrusion, southern Sinai, Egypt. J. Asian Earth Sci. 77, 203-223.

Batchelor, R.A., and Bowden, P., (1985): Petrogenetic interpretation of granitoid rock series using multicationic parameters. Chem. Geol. 48, 43-55.

Bentor, Y.K., (1985): The crustal evolution of the Arabo-Nubian Massif with special reference to the Sinai Peninsula. Precambr. Res. 28, 1-74.

Condie, K.C., (1973): Archean magmatism and crustal thickening. Geol. Soc. Am. Bull. 84, 2981-2992.

De la Roche, H., Leterrier, J., Grandclaude, P. and Marchal, M., (1980): A classification of volcanic and plutonic rocks using R1-R2 diagrams and major-element analyses-Its relation with current nomenclature. Chem. Geol., 29, 183-210.

Didier, J., Duthou, J. C. and Lameyre, J., (1982): Mantle and crust granites: genetic classification of orogenic granites and nature of their enclaves. J. Volcanol. Geotherm. Res. 14, 125-132.

EI-Sayed M. M. (1998): Tectonic setting and petrogenesis of the Kadabora pluton a late Proterozoic anorogenic A-type younger granitoid in the Egyptian Shield. Chemie der Erde, 58, 38-63.

El Mahallawi, M. M., and Ahmed, A. F., (2012): Late Proterozoic older granitoids from the North Eastern desert of Egypt: petrogenesis and geodynamic implications. Arab. J. Geosci. 5, 15-27.

El-Bialy, M. Z., and Omar, M.M., (2015): Spatial association of Neoproterozoic continental arc I type and post-collision A-type granitoids in the Arabian-Nubian Shield: the Wadi Al-Baroud older and younger granites, north Eastern Desert, Egypt. J. Afr. Earth Sci.103, 1-29. 


\section{El Afandy, et al.}

El-Bialy, M. Z., and Shata, A. E., (2018): Geochemistry, petrogenesis and radioactive mineralization of two coeval Neoproterozoic post-collisional calc-alkaline and alkaline granitoid suites from Sinai, Arabian Nubian Shield. Chemie der Erde 78, 15-39.

El-Bialy, M. Z., and Streck, M. J., (2009): Late Neoproterozoic alkaline magmatismin the ArabianNubian Shield: the postcollisional A-type granite of Sahara-Umm Adawi pluton, Sinai, Egypt. Arab. J. Geosci. 2, 151-174.

Eliwa, H. A., Breitkreuz, C., Murata, M., Khalaf, I. M., Buhler, B., Itaya, T., Takahashi, T., Hirahara, Y., Miyazaki, T., Kimura, J.-I., Shibata, T., Koshi, Y., Kato, Y., Ozawa, H., Daas, M.A., and El Gameel, Kh., (2014): SIMS zircon U-Pb and mica K-Ar geochronology, and Sr-Nd isotope geochemistry of Neoproterozoic granitoids and their bearing on the evolution of the north Eastern Desert, Egypt. Gondwana Res. 25, 1570-1598.

El-Sayed, M. M., and Nisr, S. A., (1999): Petrogenesis and evolution of the Dineibit El-Qulieb hyperaluminous leucogranite, Southeastern Desert, Egypt: petrological and geochemical constraints. J. Afr. Earth Sci. 28, 703-720.

Farahat, E. S., Mohamed, H. A., Ahmed, A. F., and El Mahallawi, M. M., (2007): Origin of I-and Atype granitoids from the Eastern Desert of Egypt: implications for crustal growth in the northern Arabian-Nubian Shield. J. Afr. Earth Sc. 49, 43-58.

Farahat, E. S., Zaki, R., Hauzenberger, C., and Sami, M., (2011): Neoproterozoic calc-alkaline peraluminous granitoids of the Deleihimmi pluton, Central Eastern Desert, Egypt: implications for transition from late- to post-collisional tectonomagmatic evolution in the northern Arabian-Nubian Shield. Geol. J. 46, 544-560.

Frost, B. R., Barnes, C. G., Collins, W. J., Arculus, R. J., Ellis, D. J., and Frost, C. D., (2001): A geochemical classification for granitic rocks. J. Petrol. 42, 2033-2048.

Gast, P.W., (1965): Terrestial ratio of Potassium and Rubidium and the composition of the Earth's mantle. Sci. 147, 858 - 860.

Hanson, G. N., (1978): The application of trace elements to the petrogenesis igneous rocks of granitic composition: Earth Planet. Sci. Lett. 38, 26-48.

Hargrove, U. S., Stern, R. J., Kimura, J. I., Manton, W. I., and Johnson, P. R., (2006): How juvenile is the Arabian-Nubian Shield? Evidence from $\mathrm{Nd}$ isotopes and pre-Neoproterozoic inherited zircon in the Bi'r Umq suture zone, Saudi Arabia. Earth Planet. Sci. Lett. 252, 308-326.

Harris, N. B. W., Pearce, J. A. and Tindle, A. G., (1986): Geochemical characteristics of collisionzone magmatism. In: Coward, M.P., Ries, A.C. (Eds.), Collision Tectonics. Geol. Soc. London Spec. Publ.,19, 67-81.

Hassan, M .A. and Hashad, A. H. (1990): Precambrian of Egypt. In: Said, R. (ed.). The geology of Egypt, Balkema, Rotterdam, 201 - 245.

Heier, K. S., (1973): A model for the composition of the deep continental crust. Fortschritte der Mineralogie 50, 174-187.

Hussein A. A, Ali M. M, El Ramly M. F. (1982): A proposed new classification of the granites of Egypt. J. Volc. Geoth. Res., 14, 187-198.

James, R. S. and Hamilton, D. L., (1969): Phase relations in the system NaAlSi3O8-KAlSi3O8$\mathrm{CaAl}_{2} \mathrm{Si}_{2} \mathrm{O}_{8}-\mathrm{SiO}_{2}$ at 1 kilobar water vapour pressure. Contributions to Mineralogy and Petrology 21, 111-141.

Jung, S., Hoernes, S. and Mezger, K., (2000): Geochronology and petrogenesis of Pan-African, syntectonic, S-type and post-tectonic A-type granite (Namibia): products of melting of crustal sources, fractional crystallization and wall rock entrainment. Lithos 50, 259-287.

Kilpatrick, J. A. and Ellis, D. J. (1992): C-type magmas: igneous charnockites and their extrusive equivalents. Trans. of the Royal Soc. Edinburgh: Earth Sci. 83, 155-164.

Laurent, O. Martina, H. Moyena, J. F. and Doucelance, R., (2014): The diversity and evolution of lateArchean granitoids: Evidence for the onset of "modern-style" plate tectonics between 3.0 and 2.5 Ga. Lithos, 205, 208-235.

Lee, S.-G., Asahara, Y., Tanaka, T., Lee, S. R. and Lee, T., (2013): Geochemical significance of the $\mathrm{Rb}-\mathrm{Sr}, \mathrm{La}-\mathrm{Ce}$ and $\mathrm{Sm}-\mathrm{Nd}$ isotope systems in A-type rocks with REE tetrad patterns and negative $\mathrm{Eu}$ and Ce anomalies: The Cretaceous Muamsa and Weolaksan granites, South Korea. Chemie der Erde, $73,75-88$. 
Geological, geochemical, and petrogenetic aspects

Liégeois, J. P. and Black, R., (1987): Alkaline magmatism subsequent to collision in the Pan- African belt of the Adrar des Iforas. In: Fitton, J.G., Upton, B.G.J. (Eds.), Alkaline Igneous Rocks. Geol. Soc. London, Spec. Publ., 30, 381-401.

Luth, W. C., Janns, R. H. and Tuttle, O. F. (1964): The granite system at pressure of 4 to 10 kilobars.J. Geophys. Res., 69, 759-773.

Mahdy, N. M., El Kaliobi, B. A., Wohlgemuth-Ueberwasser, C. C., Shalaby, M.H., and El Afandy, A. H., (2015): Petrogenesis of U- and Mo-bearing A2-type granite of the Gattar batholiths in the Arabian Nubian Shield, Northeastern Desert, Egypt: Evidence for the favorability of host rocks for the origin of associated ore deposits. Ore Geol. Rev. 71, 57-81.

Maniar, P. D. and Piccoli, P. M., (1989): Tectonic discrimination of granitoids. . Geol. Soc. Am. Bull., 101, 635-643.

Middlemost, E. A. K., (1985): Magma and magmatic rocks: An introduction to igneous petrology. Longman Inc., New York. 257p.

Moghazi, A. M., (2002): Petrology and geochemistry of Pan-African granitoids, Kab Amiri area, Egypt-implications for tectonomagmatic stages in the Nubian shield evolution. Mineral, and Petrol., 75, 41-76.

Moghazi, A. M., Hassanen, M.A., Mohamed, F.H., and Ali, S., (2004): Late Neoproterozoic strongly peraluminous leucogranites, South Eastern Desert, Egypt-petrogenesis and geodynamic significance. Mineral. Petrol. 81, 19-41.

Moussa, E. M., Stern, R. J., Manton, W. I., and Ali, K. A., (2008): SHRIMP zircon dating and Sm/Nd isotopic investigations of Neoproterozoic granitoids, Eastern Desert. Egypt. Precamb. Res., 160, 341-356.

Noweir A. M, Sewiftm BM, Abu El Ela A. M (1990): Geology, petrography, geochemistry and petrogenesis of the Egyptian younger granites. Qatar Univ Sci Bull 10, 363-393.

Pearce, J. A., (1996): Sources and settings of granitic rocks. Episodes 19, 120-125.

Pearce, J. A., Harris, N.B., and Tindle, A.G., (1984): Trace element discrimination diagrams for the tectonic interpretation of granitic rocks. J. Petrol. 25, 956-983.

Presnall, D. C. and Bateman, P. C., (1973): Function relation in the system $\mathrm{NaAlSi}_{3} \mathrm{O}_{8}-\mathrm{CaAl}_{2} \mathrm{Si}_{2} \mathrm{O}_{8}-$ $\mathrm{KAlSi}_{3} \mathrm{O}_{8}-\mathrm{H}_{2} \mathrm{O}$ and generation of the granitic magmas in the Sierra Nevada batholith. Geol. Soc. Am. Bull, 84, 3181- 3202.

Rogers, J. W. and Greenberg, J. K., (1981): Trace elements in continental margin magmatism: part 111, Alkali-granites and their relationship to cratonization: Summary. Bull. Geol. Soc. Am., 92, 6-9.

Sami, M., Ntaflos, T., Farahat, E.S., Mohamed, H.A., Hauzenberger, Ch., and Ahmed, A.F., (2018): Petrogenesis and geodynamic implications of Ediacaran highly fractionated A-type granitoids in the north Arabian-Nubian Shield (Egypt): Constraints from whole rock geochemistry. Lithos 304, 329-346.

Stern, R. J. (1979): Late Precambrian ensimatic volcanism in the Central Eastern Desert of Egypt. $\mathrm{Ph} . \mathrm{D}$. thesis, University of California, USA

Sun, S. S., and McDonough, W.F., (1989): Chemical and isotopic systematics of oceanic basalts: implications for mantle composition and processes. Geol. Soc. London, Spec. Publ. 42, 313-345.

Taylor, S. R., (1965): The application of trace element data to problems in petrology. Phys. Chem. Earth, 6, 133-213.

Taylor, S. R., Emeleus, C. H. And Exley, C. S., (1956): Some anomalous K/Rb ratios in igneous rocks and their petrological significance. Geochim. Cosmochim. Acta, 10, 224 - 229.

Tuttle, O. F., and Bowen, N. L., (1958): Origin of granite in the light of experimental studies in the system $\mathrm{NaAlSi}_{3} \mathrm{O}_{8}-\mathrm{SiO}_{2}-\mathrm{H}_{2} \mathrm{O}$. Geol. Soc. Am. Mem., 74, 153.

Whalen, J.B., Currie, K.L., and Chappell, B.W., (1987): A-type granites: geochemical characteristics, discrimination and petrogenesis. Contrib. Miner. Petrol. 95, 407-419.

White, A.J.R. and Chappell, B.W., (1983): Granitoid types and their distribution in the Lachlan Fold Belt, southeastern Australia. In: Roddick, J.A. (Ed.), Circum-Pacific Plutonic Terranes. Geol. Soc. Am., Memior 159, 21- 34.

Whitney, J. A., Jones, L. M. and Walker, R. L., (1976): Age and origin of the Stone Mountain granite, Lithonia District, Georgia. . Geol. Soc. Am. Bull., 87, 1067-1077.

Winkler, H. G. F., Boese, M. and Marcoulos, T., (1975): Low temperature granitic melts. Neues Jahrbuch für Mineralogie Monatsh efte 6, 245-68.

Zen, E.-An., (1986): Aluminum enrichment in silicate melts by fractional crystallization: some mineralogic and petrographic constraints. J. Petrol., 27, 1095-1117. 
El Afandy, et al.

الجوانب الجيولوجية والجيوكيميائية والتكوينية لجرانيتات النيويروتيروزيك الحديثة بمنطقة وادي أم سيدرا ووادي أم

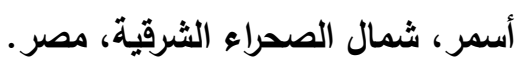

عادل حسن الافندي'و باهر عبد الحميد القليوبي'و حسن علي عليوة'ّو حسام انور خميس' وصديق حمدي صديق' ا ـ هيئة المواد النووية، ص.ب . •rه ، المعادي، القاهرة، مصر •

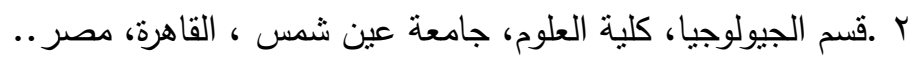

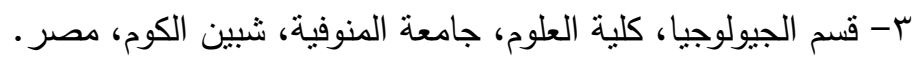

\section{الخلاصة}

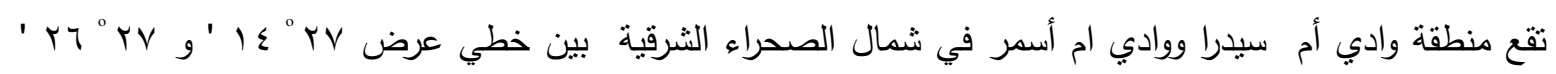

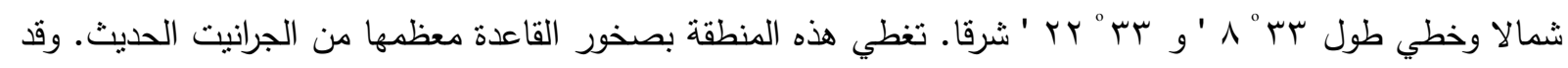

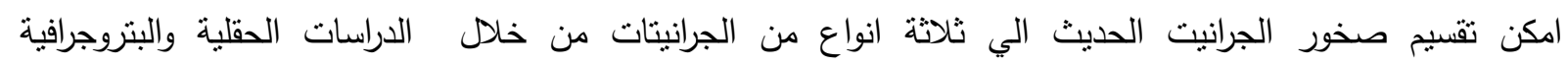
والجيوكيميائية وهي المونزوجرانيت و السيانوجرانيت و جرانيت الفلسبار القلوي و كل نوع من الجرانيت له خصائصنه الخاصة به. توجد صخور المونزوجرانيت علي هيئة نالا منخفضة الي متوسطة الارتفاع وتتمنل في جبل ابو المساعيد ونيد ويخترق هذا الجرانيت صخور بركانيات الاخان. اما صخور السيانوجرانيت تتواجد في جبل ابو حربة علي هيئة ارتفاعات

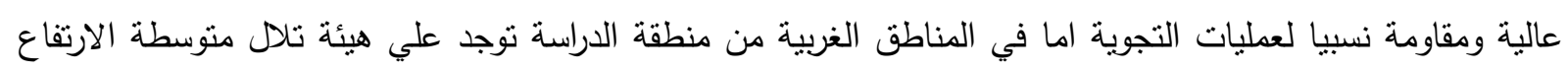
ويخترق هذا الجرانيت صخور بركانبات الدخان والبركانيات المتحولة. توجد صخور جرانيت الفلسبار القلوي علي هئئية احزمة جبلية تمند من جبل الحروث في الجنوب الي جبل الشعيلة في الثمال بارتفاعات عالية مقاومة لعمليات التجوية

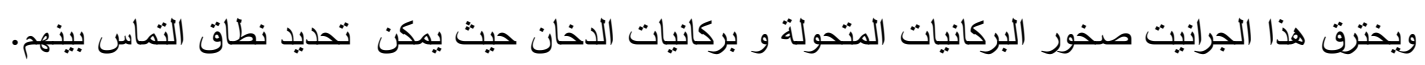
وقد اوضحت الدراسات البتروجرافية لهذة الجرانيتات ان معادن الفلسبار القلوي والكوارتز تزداد من المونزوجرانيت مرورا

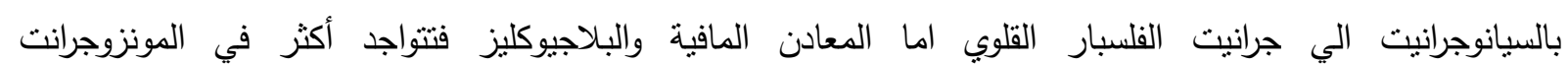
والسيانوجرانيت. بالنوجران.

والقد اكدت الدراسة الجيوكيميائية لصخور المونزوجرانيت والسيانوجرانيت انها نشأت من صهارة كلسية قلوية في حين

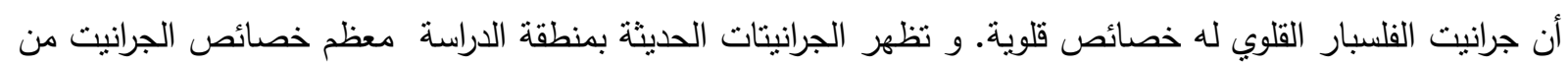

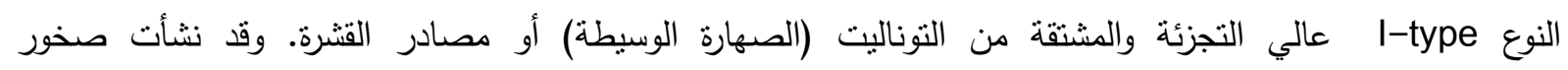

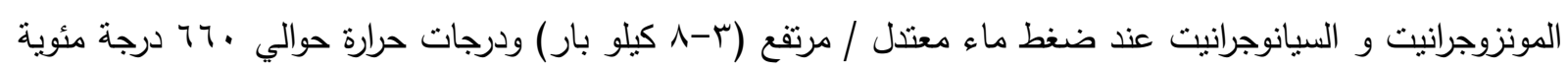



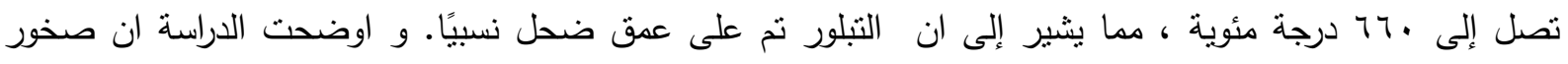

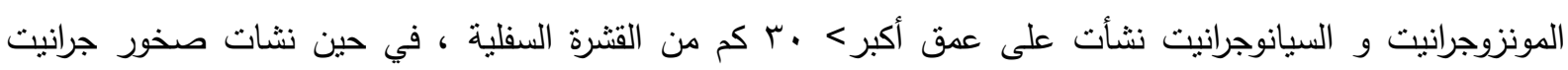

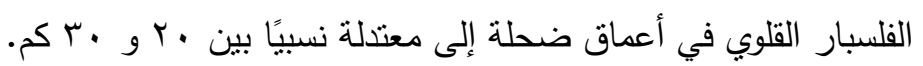

